\title{
SMARCA4 regulates gene expression and higher- order chromatin structure in proliferating mammary epithelial cells
}

\author{
A. Rasim Barutcu, ${ }^{1}$ Bryan R. Lajoie, ${ }^{2}$ Andrew J. Fritz, ${ }^{3}$ Rachel P. McCord, ${ }^{4}$ \\ Jeffrey A. Nickerson, ${ }^{1}$ Andre J. van Wijnen, ${ }^{5}$ Jane B. Lian, ${ }^{3}$ Janet L. Stein, ${ }^{3}$ Job Dekker, ${ }^{2,6}$ \\ Gary S. Stein, ${ }^{3}$ and Anthony N. Imbalzano ${ }^{1}$ \\ ${ }^{1}$ Department of Cell and Developmental Biology, University of Massachusetts Medical School, Worcester, Massachusetts 01655 , \\ USA; ${ }^{2}$ Program in Systems Biology, University of Massachusetts Medical School, Worcester, Massachusetts 01605, USA; \\ ${ }^{3}$ Department of Biochemistry, University of Vermont College of Medicine, Burlington, Vermont 05405, USA; ${ }^{4}$ Department of \\ Biochemistry \& Cellular and Molecular Biology, University of Tennessee, Knoxville, Tennessee 37996, USA; ${ }^{5}$ Department of \\ Biochemistry and Molecular Biology, Mayo Clinic, Rochester, Minnesota 55905, USA; ${ }^{6}$ Howard Hughes Medical Institute, Department \\ of Biochemistry and Molecular Pharmacology, University of Massachusetts Medical School, Worcester, Massachusetts 01605, USA
}

\begin{abstract}
The packaging of DNA into chromatin plays an important role in transcriptional regulation and nuclear processes. Brahmarelated gene-1 SMARCA4 (also known as BRG1), the essential ATPase subunit of the mammalian SWI/SNF chromatin remodeling complex, uses the energy from ATP hydrolysis to disrupt nucleosomes at target regions. Although the transcriptional role of SMARCA4 at gene promoters is well-studied, less is known about its role in higher-order genome organization. SMARCA4 knockdown in human mammary epithelial MCF-10A cells resulted in 176 up-regulated genes, including many related to lipid and calcium metabolism, and 1292 down-regulated genes, some of which encode extracellular matrix (ECM) components that can exert mechanical forces and affect nuclear structure. ChIP-seq analysis of SMARCA4 localization and SMARCA4-bound super-enhancers demonstrated extensive binding at intergenic regions. Furthermore, $\mathrm{Hi}-\mathrm{C}$ analysis showed extensive SMARCA4-mediated alterations in higher-order genome organization at multiple resolutions. First, SMARCA4 knockdown resulted in clustering of intra- and inter-subtelomeric regions, demonstrating a novel role for SMARCA4 in telomere organization. SMARCA4 binding was enriched at topologically associating domain (TAD) boundaries, and SMARCA4 knockdown resulted in weakening of TAD boundary strength. Taken together, these findings provide a dynamic view of SMARCA4-dependent changes in higher-order chromatin organization and gene expression, identifying SMARCA4 as a novel component of chromatin organization.
\end{abstract}

[Supplemental material is available for this article.]

\begin{abstract}
Organization of chromatin is essential for many biological processes. Packaging of the DNA around the nucleosomes acts to tightly condense the genome (Cutter and Hayes 2015). At the same time, the cell has to regulate the accessibility of the chromatin to many enzymes for the regulation of gene expression, DNA replication, and repair. Maintaining a balance between tight packaging and accessibility of the chromatin is an important function of the eukaryotic nucleus. This balance is achieved by multiple specialized protein complexes that dynamically alter chromatin structure in an ATP-dependent manner (Clapier and Cairns 2009). Four families of ATP-dependent chromatin remodelers exist: SWI/SNF, ISWI, INO80, and CHD (for review, see Varga-Weisz 2001; Flaus and Owen-Hughes 2011). The ATPase subunits of each family have a conserved helicase-like ATPase domain that uses the energy from ATP hydrolysis to evict, reposition, or modify nucleosomes (Sala et al. 2011; Narlikar et al. 2013). Different families of remodelers work in cells in a dynamic and orchestrated way to fine tune DNA accessibility (Morris et al. 2014).
\end{abstract}

Corresponding authors: gary.stein@uvm.edu, anthony.imbalzano@ umassmed.edu

Article published online before print. Article, supplemental material, and publication date are at http://www.genome.org/cgi/doi/10.1101/gr.201624.115.
The mammalian mating-type switching and sucrose nonfermenting (SWI/SNF) complexes contain one of two distinct ATPase subunits, SMARCA2 (also known as Brahma [BRM]) or SMARCA4 (Muchardt and Yaniv 1993; Wang et al. 1996). SMARCA2 is thought to be dispensable, as SMARCA2 null mice can properly develop to adulthood (Reyes et al. 1998), although this finding has recently been questioned (Thompson et al. 2015). On the other hand, SMARCA4 is essential, as SMARCA4 null mice are embryonic lethal, and SMARCA4 heterozygous mice show developmental defects and are prone to mammary tumor formation (Bultman et al. 2000, 2008). SMARCA4 has been shown to be involved in many developmental processes and in transcriptional regulation, DNA repair, cell cycle control, and cancer (Trotter and Archer 2008; King et al. 2012). The role of SMARCA4 in gene regulation is contextual, as it can activate some promoters while repressing others. In addition, extensive dysregulation and mutations of SMARCA4 have been implicated

(c) 2016 Barutcu et al. This article is distributed exclusively by Cold Spring Harbor Laboratory Press for the first six months after the full-issue publication date (see http://genome.cshlp.org/site/misc/terms.xhtml). After six months, it is available under a Creative Commons License (Attribution-NonCommercial 4.0 International), as described at http://creativecommons.org/licenses/ by-nc/4.0/. 
in many different cancer types, making SMARCA4 a potential therapeutic target for cancer (Kadoch et al. 2013; Shain and Pollack 2013).

Through interactions with many different protein partners, SMARCA4 is involved in nuclear structure and in mediating specific long-range chromatin interactions (Trotter and Archer 2008; Euskirchen et al. 2011; Imbalzano et al. 2013a). The organization of chromatin occurs in a hierarchical manner. Chromosomes are positioned in distinct volumes forming the chromosome territories (Cremer et al. 2006), which consist of open (A-type) and closed (Btype) genomic compartments (Lieberman-Aiden et al. 2009). The genomic compartments are further folded into sub-megabasescaled structures called topologically associating domains (TADs) (Dixon et al. 2012; Nora et al. 2012, 2013), where local looping interactions between promoters and enhancers occur (Sanyal et al. 2012; Symmons et al. 2014; Tang et al. 2015; Smith et al. 2016).

SMARCA4 regulates inter-chromosomal interactions between tissue-specific promoters during myogenesis (Harada et al. 2015) and is required for looping at many gene loci, including the beta and alpha-globin genes (Kim et al. 2009a,b), the IgH locus (Bossen et al. 2015), and the class II major histocompatibility complex gene (CIITA) (Ni et al. 2008). SMARCA4 binds to poised developmental enhancers in embryonic stem cells (Hu et al. 2011; RadaIglesias et al. 2011) and B-cells (Bossen et al. 2015) and colocalizes with pluripotency factors (Ho et al. 2009), suggesting important roles in enhancer function. Furthermore, previous work classifying genome-wide interactions according to their histone modifications and transcription factor binding revealed SMARCA4 enrichment at open chromatin regions, indicating a possible structural role for SMARCA4 (Lan et al. 2012). In addition, SMARCA4 knockdown affects nuclear size (Hill et al. 2004) and the integrity of nuclear shape via a mechanism independent of cytoskeletal connections (Imbalzano et al. 2013b). Recently, SMARCA4 was shown to be involved in the IncRNA-dependent assembly of nuclear bodies (Kawaguchi et al. 2015).

Taken together, apart from its chromatin remodeling activity at the regulatory regions of target genes, emerging evidence suggests a possible important role for SMARCA4 in maintaining the structural integrity of the nucleus by regulating global chromatin structure (Imbalzano et al. 2013a). To date, very little is known about the role of SMARCA4 in global higher-order genome organization. To gain insight into the role of SMARCA4 in nuclear organization at a genome-wide level, we performed RNA-seq and Hi-C in SMARCA4-knockdown and control MCF-10A human mammary epithelial cells. Furthermore, to map the localization of SMARCA4 in the genome, we performed SMARCA4 ChIP-seq in the parental MCF-10A cells. Overall, we show that SMARCA4 knockdown is associated with extensive changes in gene expression and higher-order chromatin structure, affecting TAD boundaries and telomere clustering.

\section{Results}

\section{SMARCA4 globally affects gene regulation in MCF-10A cells}

To investigate the roles of SMARCA4 in transcriptional regulation, we used previously described doxycycline-inducible MCF10A mammary epithelial cells expressing either a nonspecific (scrambled) shRNA (shSCRAM), or shRNA against SMARCA4 (shSMARCA4) (Cohet et al. 2010). We confirmed the down-regulation of the SMARCA4 protein by Western blot analysis in doxycycline-induced cells (Fig. 1A).
Next, we performed poly(A) RNA-seq in doxycycline-induced shSMARCA4 and shSCRAM MCF-10A cells (Supplemental Figs. S1A-E, S2A,B). We identified 176 up-regulated and 1292 down-regulated genes upon SMARCA4 knockdown (Fig. 1B,C; Supplemental Table S1). REACTOME pathway analysis (Milacic et al. 2012; Croft et al. 2014) of down-regulated genes identified pathways related to "extracellular matrix (ECM) organization," "collagen degradation," "cell adhesion molecule L1-like (CHL1) interactions," and "cohesin loading onto chromatin" (Fig. 1D). A significant number of these genes were associated with cell adhesion, including many proteoglycans, integrins, and laminins. This result suggests a role for SMARCA4 in ECM biology and nuclear structure. On the other hand, up-regulated genes were associated with calcium signaling and lipid metabolism, including pathways related to "regulation of cholesterol biosynthesis by SREBP," "fatty acids," and "eicosanoids," which are the byproducts of fatty acid oxidation (Fig. 1E).

Furthermore, we assessed the transcriptional changes of polyadenylated long noncoding RNAs (lncRNAs) using the GENCODE v19 lncRNA gene annotation (Trapnell et al. 2013). We identified 88 down-regulated and 64 up-regulated poly(A) lncRNAs upon SMARCA4 knockdown (Supplemental Table S2). This suggests a widespread role of SMARCA4 in the transcriptional regulation of both coding and noncoding genes. Although the majority of the differentially expressed IncRNAs were unannotated, we observed down-regulation of several well-known lncRNA genes, including XIST, NEAT1, and MALAT1, and up-regulation of the imprinted IncRNA H19. We validated the RNA-seq results by performing qRT-PCR on 23 coding and noncoding genes of interest. There was a significant correlation between qRT-PCR and RNA-seq results (Spearman's $\rho=0.65, P=4.17 \times 10^{-6}$ ) (Supplemental Fig. S2C,D).

Taken together, these results suggest that SMARCA4 positively regulates genes associated with ECM, supporting the role for SMARCA4 in ECM biology (Xu et al. 2007; Stankunas et al. 2008; Saladi et al. 2010), and negatively regulates genes related to lipid metabolism and calcium signaling in mammary epithelial cells. Moreover, SMARCA4 also regulates the expression of many lncRNAs.

\section{SMARCA4 ChIP-seq analysis reveals extensive binding to intergenic and intronic regions in MCF-10A cells}

To confirm that SMARCA4-dependent alterations in gene expression observed by RNA-seq are directly related to SMARCA4 binding, we performed ChIP-seq analysis in wild-type MCF-10A cells (Fig. 2; Supplemental Fig. S3A,B). We identified 15,046 SMARCA4 ChIP-seq peaks. The binding profile of SMARCA4 demonstrated that SMARCA4 binds to defined locations in the genome (Fig. 2A). Annotation of SMARCA4 ChIP-seq peaks revealed that $60 \%$ of the binding sites were localized in promoters, introns, and exons (gene bodies), whereas $40 \%$ of the sites were bound to intergenic regions (Fig. 2B). Consistent with the annotation of SMARCA4 peaks, normalized SMARCA4 binding signal across all human genes was highest at promoter regions (Fig. 2C). Because SMARCA4 was enriched at the promoters, we intersected the SMARCA4 peaks with a publicly available MCF-10A Pol II ChIPseq data set (GSM935588) (The ENCODE Project Consortium 2012) and determined that $27 \%$ of all SMARCA4 bound sites were also bound by Pol II (Supplemental Fig. S3C).

By performing de novo motif analysis on SMARCA4 peaks, we identified enriched motifs for MEF2A/C, USF2, SMAD2/4, TP53, and SPI1 (Fig. 2D). These factors may be functionally related 
A
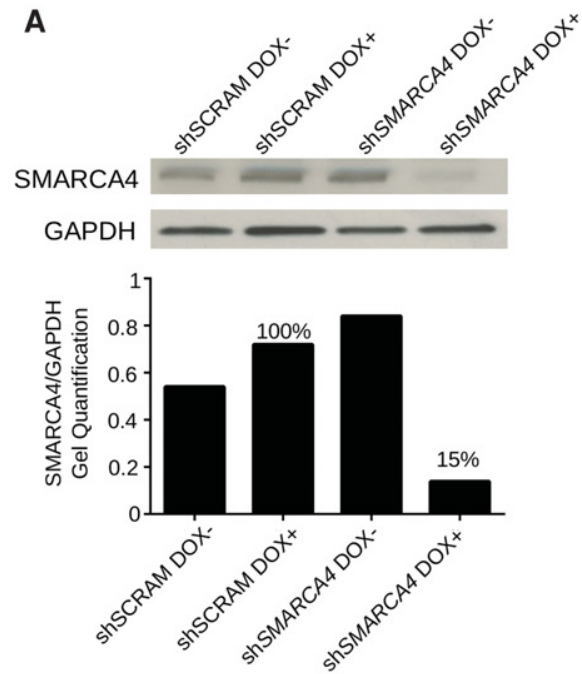

C

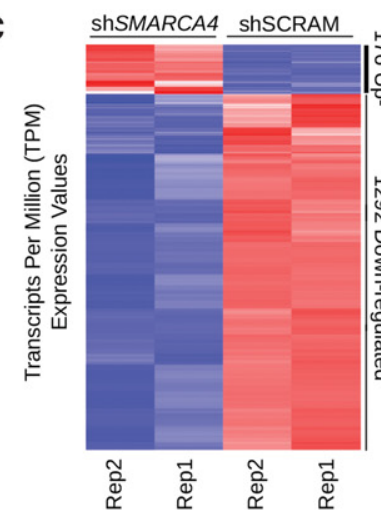

ShSMARCA4/shSCRAM 今ั $\quad \log 2$ Fold Change

D

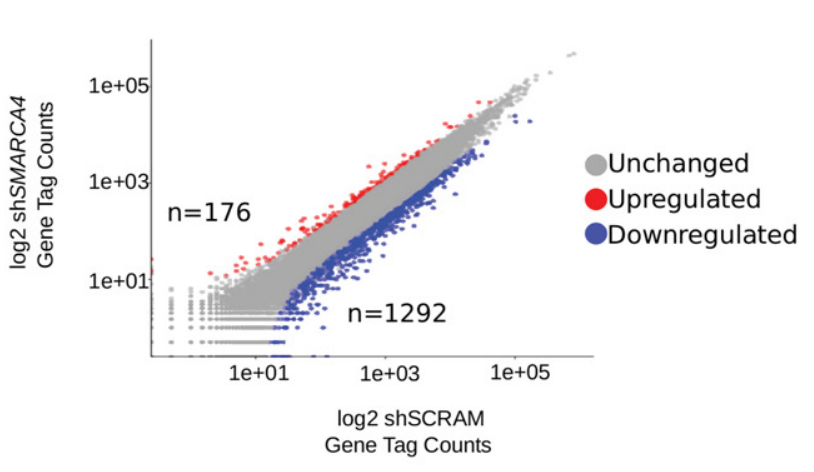

B

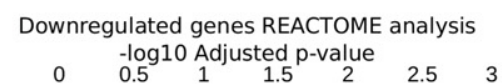

Fibronectin matrix formation $0 \quad 0.5 \quad 1 \quad 1.5 \quad 2$

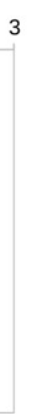

E

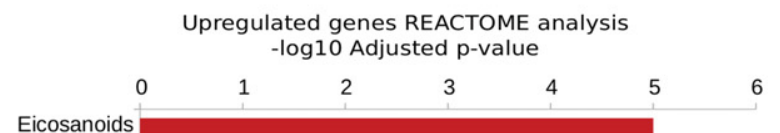
Activation of gene expression by SREBF (SREBP) Regulation of cholesterol biosynthesis by SREBP (SREBF)

Fatty acids Cam-PDE 1 activation Adenosine $\mathrm{P} 1$ receptors GRB7 events in ERBB2 signaling Miscellaneous substrates Synthesis of Leukotrienes (LT) and Eoxins (EX) Cytochrome P450 - arranged by substrate type Calmodulin induced events CaM pathway Molecules associated withelastic fibres

Extracellular matrix organization

Cohesin Loading onto Chromatin

$\mathrm{CHL} 1$ interactions
Collagen degradation

Elastic fibre formation
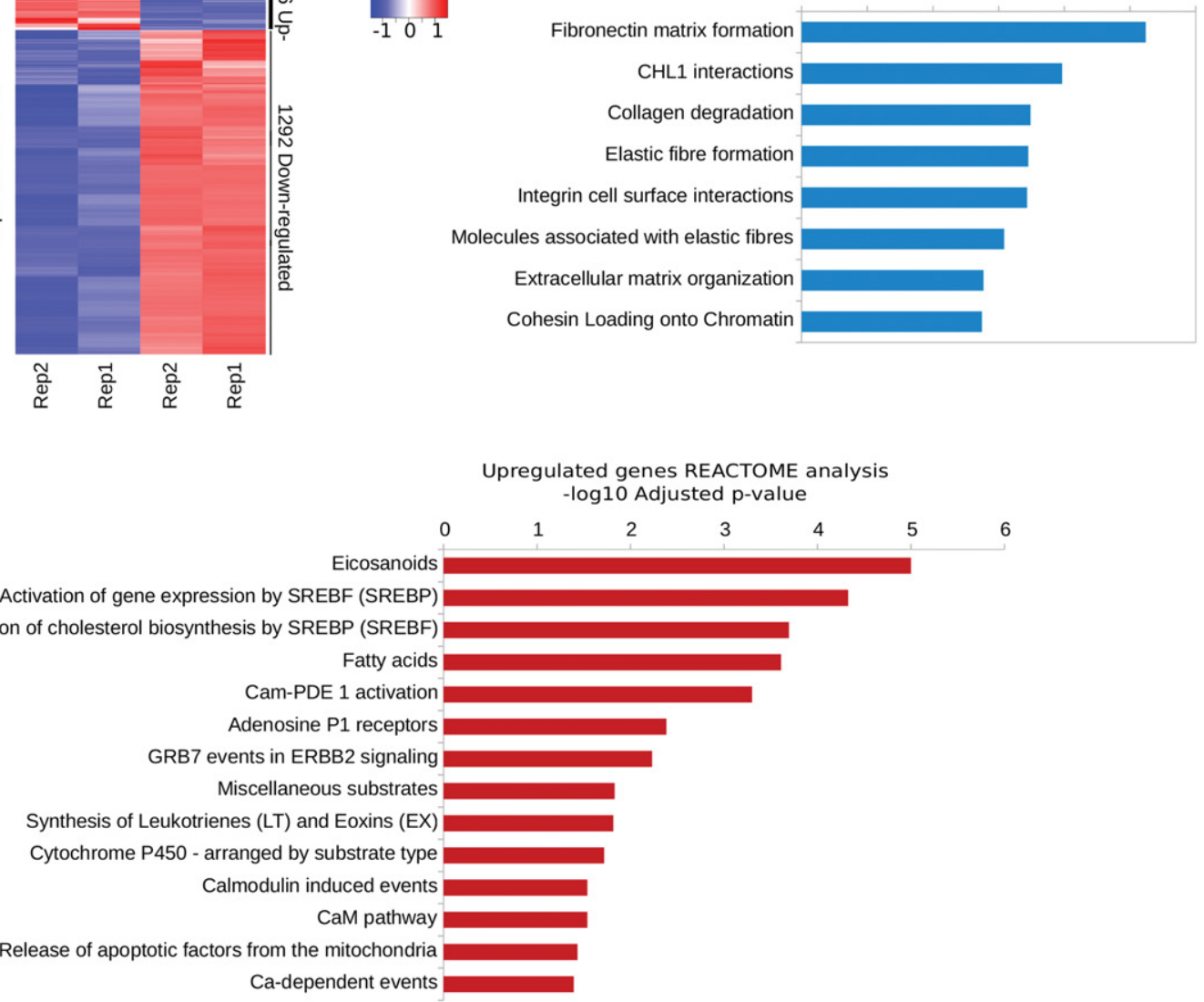

Figure 1. (A) Western blot of the SMARCA4 protein levels of shSCRAM and shSMARCA4 MCF-10A cells in the noninduced (DOX-) and induced (DOX+) conditions. Lower: Quantification of the Western blot showing $\sim 85 \%$ reduction of SMARCA4 protein levels upon doxycyline induction. (B) Scatter plot showing the $\log _{2}$ gene expression values for shSMARCA4 and shSCRAM cells. The red and blue dots denote the up- and down-regulated genes between the two conditions, respectively. (C) Heatmap showing the transcripts per million (TPM) expression values of the differentially expressed genes between shSCRAM and shSMARCA4 for each biological replicate. $(D, E)$ Bar graphs showing the $-\log _{10} P$-values for the REACTOME terms of the $(D) 1292$ genes that are down-regulated and $(E) 176$ up-regulated genes upon SMARCA4 knockdown.

because SMARCA4 is a coregulator for many of these factors. MEF2C has been shown to interact with SMARCA4 and is required for the activity-dependent recruitment of SMARCA4 to its target regions (Zhang et al. 2015). Moreover, USF1/2 can bind a SMARCA4-associated factor, SMARCD3, and recruit other BAF subunits including SMARCA4 (Wang and Sul 1997). In addition, 


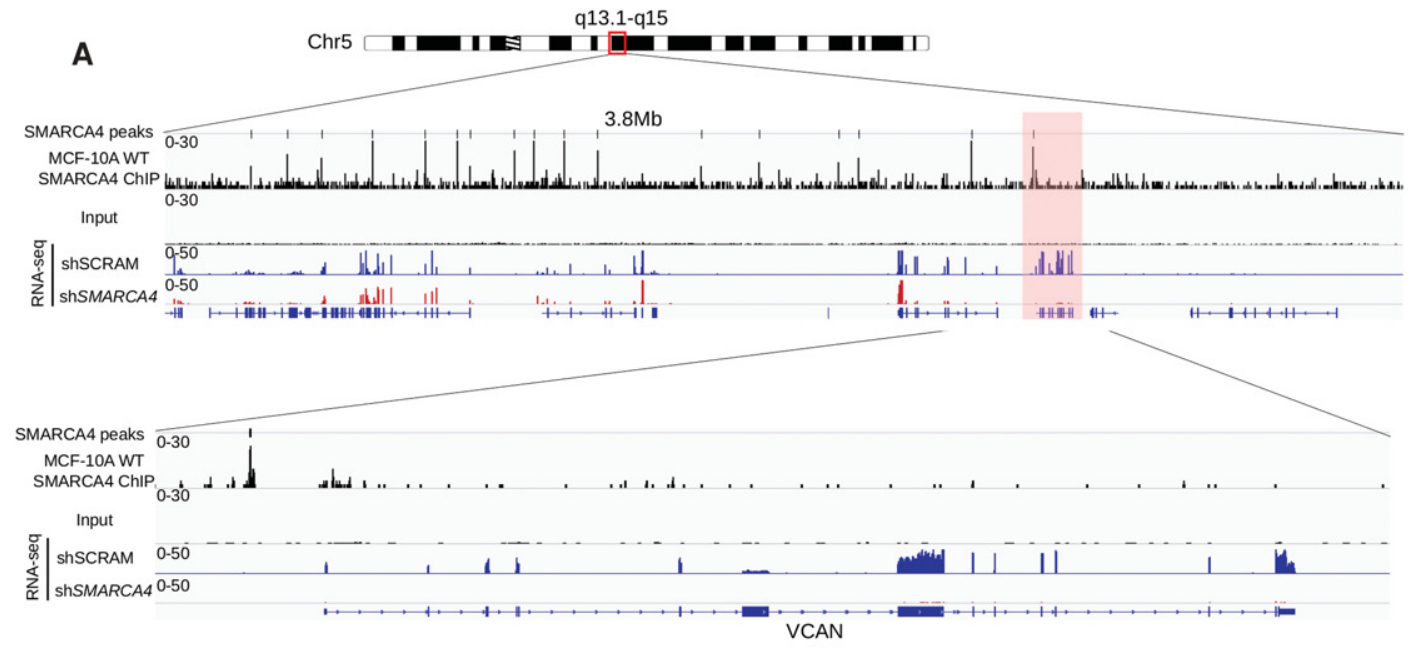

B SMARCA4 Sites $(15,046)$
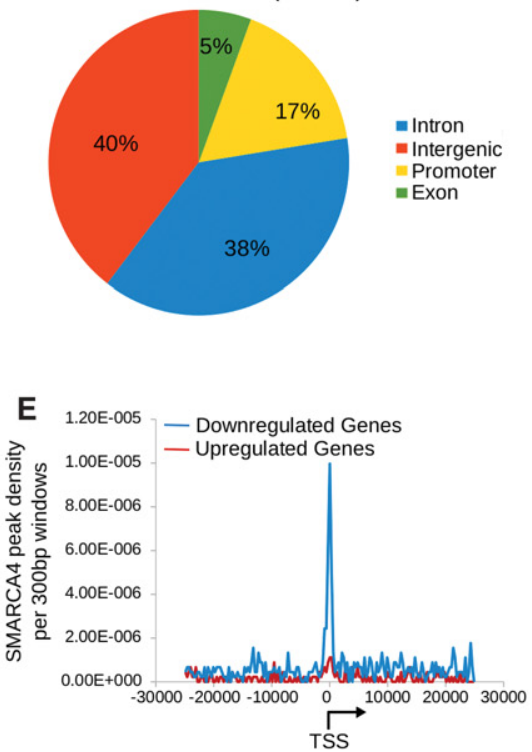

G



C

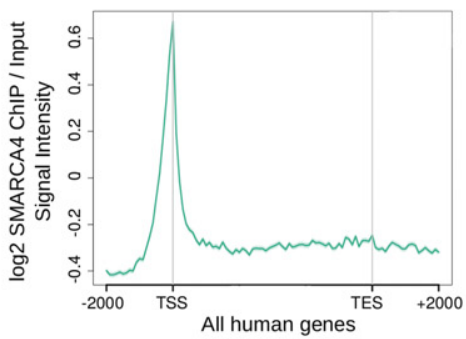

F

H
D

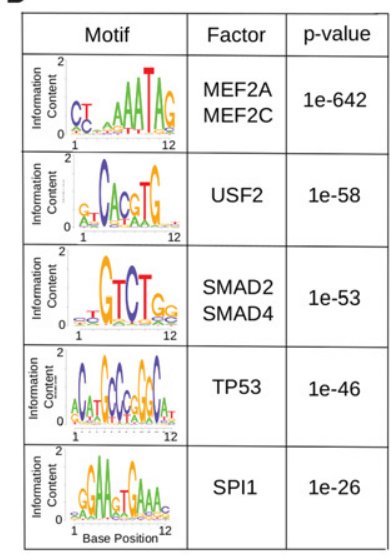

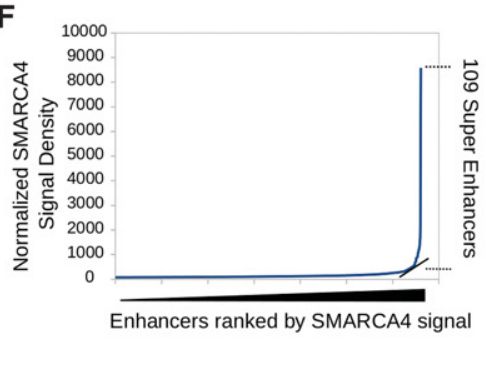

SMARCA4 Super Enhancers (109)

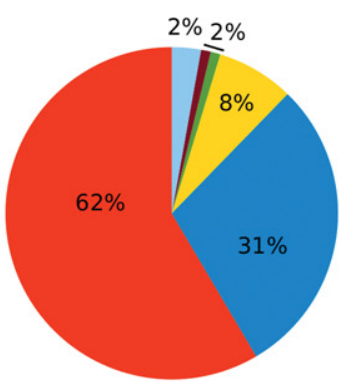

= Intron

- Intergenic

TTS

- 3'UTR

- 5 'UTR
Exon

Figure 2. (A) Example of a ChIP-seq genome browser view of SMARCA4 binding and the input control, the shSCRAM and shSMARCA4 RNA-seq on Chr 5 , and a zoom-in on the VCAN (Versican) gene, which is regulated by SMARCA4, in the lower panel. The $y$-axis represents the normalized tag densities relative to hg19 genomic coordinates. (B) Distribution of SMARCA4 ChIP-seq peak annotation for genic and intergenic regions. (C) Normalized SMARCA4 ChIPseq signal intensity plot for all human UCSC genes $\pm 2 \mathrm{~kb}$. SMARCA4 binding is enriched at the promoter regions. (D) Top five sequence motifs associated with the SMARCA4 peaks. (E) SMARCA4 peak density within $\pm 20 \mathrm{~kb}$ of the TSS of significantly down-regulated (blue), or up-regulated genes (red). ( $F$ ) Distribution of SMARCA4 ChIP-seq signal across the MCF-10A enhancers. SMARCA4 binding is not uniformly distributed across the enhancers, as 109 super-enhancers display higher $\left(\log _{2} \sim 1.5\right.$-fold) levels of SMARCA4 binding. (G) SMARCA4 signal is greater over super-enhancers (red) than typical enhancers (green). $(H)$ Distribution of SMARCA4-bound super-enhancers in genic and intergenic regions. 
SMARCA4 is a direct interaction partner for SMAD proteins and TP53 (Xi et al. 2008; Naidu et al. 2009).

Next, we asked whether SMARCA4 binding was associated with differentially expressed genes. To address this, we analyzed the SMARCA4 peak frequency at the promoters of up- and downregulated genes. We observed $\sim 10$-fold increase in the frequency of SMARCA4 binding at the promoters of genes that are down-regulated upon SMARCA4 knockdown, whereas there was minimal association of SMARCA4 with the promoters of genes that are up-regulated when SMARCA4 is knocked down (Fig. $2 \mathrm{E})$. This result suggests a more direct role for SMARCA4 in the positive regulation of gene expression in MCF-10A cells.

Recently, super-enhancers, a novel type of regulatory regions having an unusual enrichment of transcription factors that are in close proximity, were described (Whyte et al. 2013). Super-enhancers are mostly associated with developmentally regulated genes. SMARCA4 is localized at super-enhancers in leukemic and in normal B-cells (Shi et al. 2013; Bossen et al. 2015). Using the approach previously published (Whyte et al. 2013), we identified 109 SMARCA4-bound superenhancers in the MCF-10A genome (Fig. 2F). SMARCA4 signal intensity was greater at super-enhancers than at typical SMARCA4 ChIP-seq peak regions (Fig. $2 \mathrm{G})$. Annotation of the super-enhancers revealed $\sim 60 \%$ localization at intergenic regions and $\sim 40 \%$ localization at gene bodies, but not at promoters (Fig. 2H).

Overall, we identified SMARCA4 binding mostly (60\%) at promoters and gene bodies. In the promoter regions, there may be individual single (standalone) high-density SMARCA4 peaks, but since they are not proximal to other high-density SMARCA4 peaks, they are not defined as super-enhancers. This result may explain why the super-enhancers are mostly (60\%) enriched at intergenic regions, whereas the typical SMARCA4 ChIP-seq peaks are mostly (60\%) at promoters and gene bodies. Interestingly, the localization pattern of SMARCA4-bound super-enhancers is opposite of the annotation of typical SMARCA4 peaks (intergenic versus promoter) (Fig. 2B), suggesting differential regulatory functions for regular SMARCA4 peaks and SMARCA4-bound super-enhancers.

\section{Hi-C analysis of SMARCA4 knockdown and control MCF-10A cells}

To identify the genome-wide changes in higher-order chromatin structure following SMARCA4 knockdown, we performed Hi-C on doxycycline-induced shSCRAM and shSMARCA4 MCF-10A cells (Fig. 3A,B; Supplemental Table S3). With an average depth of approximately 115 million reads per biological replicate and

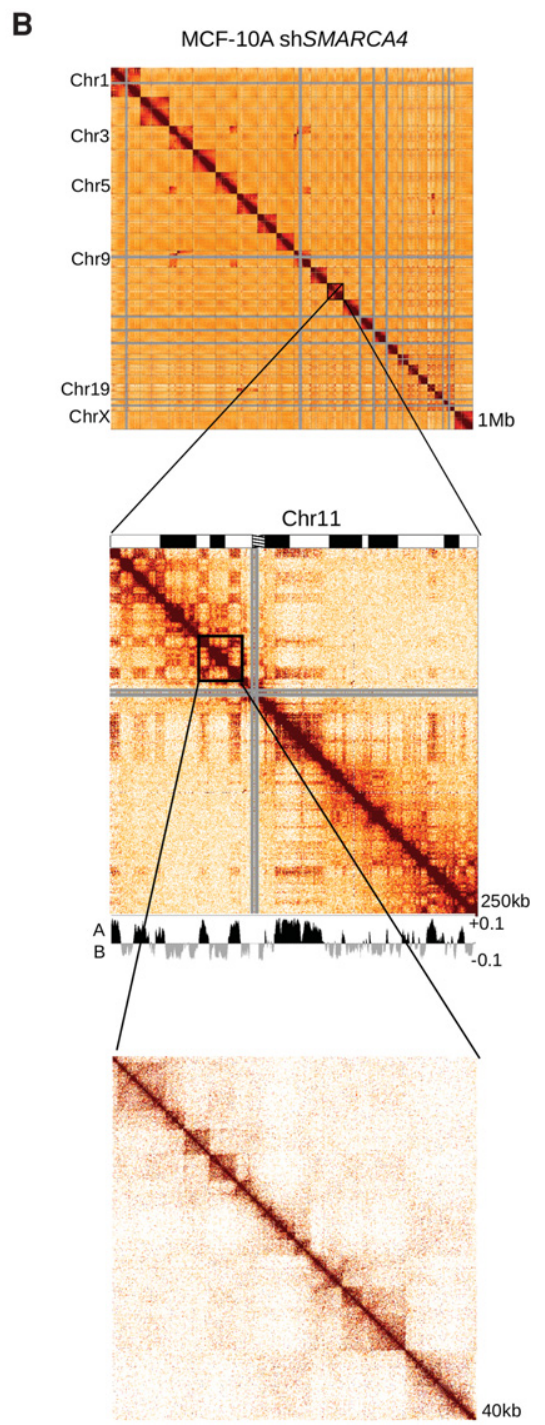

Genome-wide all-by-all $\mathrm{Hi}-\mathrm{C}$ interaction heatmaps at 1-Mb resolution and a zoom-in of $\mathrm{Chr}$ 11 at 250-kb resolution (middle) and at 40-kb resolution (lower) in MCF-10A shSCRAM $(A)$ and MCF-10A ShSMARCA4 cells (B). For the genome-wide heatmaps, the chromosomes are stacked from top-left to bottom-right in order (Chr 1, Chr 2, ..., Chr 22, and Chr X). The gray regions indicate repetitive regions (such as centromeres) in which the sequencing reads could not be mapped. The genomic compartments are shown below the middle heatmaps.

with two biological replicates, we achieved up to 40-kb resolution genome-wide. The Hi-C heatmaps, which are normalized by total number of reads, revealed hierarchical higher-order chromatin structures, such as genomic compartments and TADs, at increasing resolutions (Fig. 3A,B). There was a high correlation $\left(R^{2}>0.90\right)$ between two independent biological replicates at multiple resolutions (Supplemental Fig. S4A-J). The shSCRAM and shSMARCA4 Hi-C data sets exhibited high correlation $\left(R^{2}>0.95\right)$ with the previously published wild-type MCF-10A Hi-C data set (Barutcu et al. 2015). The scaling plot curves of genomic interaction frequencies along genomic distance showed similar trends of decay and an increase at distances larger than $>200 \mathrm{Mb}$, which we previously showed to be a characteristic of the MCF-10A genome (Supplemental Fig. S4C; Barutcu et al. 2015). The Hi-C data sets displayed similar cis/trans interaction ratios (an average of $\sim 25 \%$ ) (Supplemental Fig. S4K,L).

\section{Genome Research}

www.genome.org 
Genome-wide Hi-C interaction heatmaps showed that, consistent with previous Hi-C studies and the notion of chromosome territories (Cremer et al. 2006), the intra-chromosomal interactions, which are visualized as dark boxes along the diagonal, were more frequent than inter-chromosomal interactions (Fig. 3). Moreover, we identified large blocks of inter-chromosomal interactions between Chr 3 and Chr 5, Chr 3 and Chr 9, and Chr 6 and Chr 19, which represent previously known translocations in the MCF-10A genome (Supplemental Fig. S5; Marella et al. 2009; Barutcu et al. 2015). Taken together, these analyses reflect the high quality and reproducibility of the Hi-C data.

\section{SMARCA4 knockdown results in extensive gain and loss of long-range chromatin interactions and altered telomeric associations}

To map the SMARCA4-mediated alterations in higher-order chromatin structure, we compared the genome-wide interactions as previously described (Fig. 4A; Barutcu et al. 2015; Crane et al. 2015). At multiple resolutions (10-Mb to $40-\mathrm{kb}$ resolution genome-wide), SMARCA4 knockdown resulted in the disruption of existing interactions and the emergence of novel specific contacts throughout each chromosome (Fig. 4B; Supplemental Fig. S6). Mapping the high-confidence interactions that are depleted/enriched upon SMARCA4 knockdown revealed specific regions potentially important for transcriptional regulation (Fig. 4D,E). For instance, a zoom-in view of the differential interactions at the promoter regions of MALAT1 and NEAT1 lncRNAs, which are downregulated upon SMARCA4 knockdown, displayed several differentially interacting regions (Fig. 4D,E).

Although there were several alterations in long-range interactions at high resolution, the positioning of chromosomes relative to each other was similar following SMARCA4 knockdown (Supplemental Fig. S7A,B). At the chromosomal scale, when only the significant differential interactions were considered, SMARCA4 knockdown resulted in a higher frequency (Wilcoxon ranksum test, $P<1 \times 10^{-180}$ ) of trans interactions between the chromosomes (Supplemental Fig. S7C). Moreover, SMARCA4 knockdown resulted in a lower frequency (Wilcoxon ranksum test, $P<1.2 \times 10^{-106}$ ) of cis interactions both within and across the different chromosomal arms on each chromosome (Supplemental Fig. S7D).

Interestingly, among the subtelomeric regions of the chromosomes, we observed a systematic pattern of increased interactions in shSMARCA4 cells, both in cis and in trans (Fig. 4F). In other words, the subtelomeric regions of each chromosome displayed a striking enrichment of interactions with each other upon SMARCA4 knockdown compared with the shSCRAM control (Fig. 4F). Quantification of the subtelomeric interactions suggested a significant increase in both intra- and inter-chromosomal associations in shSMARCA4 cells compared to shSCRAM (Fig. 4G). However, this was not the case when interactions in the same regions were randomized (Supplemental Fig. S7E). To validate the Hi-C results, we performed DNA-FISH by probing the intra-chromosomal telomeric interactions of Chr 1 and $\mathrm{Chr} 4$. Consistent with our Hi-C findings, SMARCA4 knockdown resulted in a significant increase in telomeric proximity of the $\mathrm{p}$ and the $\mathrm{q}$ chromosomal arms of Chr 1 and Chr 4 (Fig. 4H,I). Taken together, these results indicate a novel role for SMARCA4 in telomere structure and suggest that disruption of SMARCA4 levels results in altered three-dimensional organization of telomeric regions of the genome.

\section{SMARCA4 occupancy is enriched at open compartment regions}

Each chromosome territory is composed of megabase-scale genomic compartments that are either A-type (i.e., open, gene rich) or B-type (i.e., closed, gene poor). The frequency of interactions within one compartment is greater than the frequency of interactions between compartments (Lieberman-Aiden et al. 2009). We asked whether SMARCA4 knockdown resulted in any compartment changes. To address this, we binned the genome at $250-\mathrm{kb}$ nonoverlapping intervals and compared the type of compartmentalization for each bin (Fig. 5A). The majority of the compartmentalization was similar in shSCRAM and shSMARCA4 MCF-10A cells, with $42 \%$ of the genome consisting of constitutive A-type compartments and $54 \%$ consisting of constitutive B-type compartments (Fig. 5B). Upon SMARCA4 knockdown, a total of $2 \%$ of the genome altered its compartmentalization from A-type to B-type and $2 \%$ showed alteration from B-type to A-type (Fig. 5B).

Compartmentalization of the genome is correlated with gene expression (Barutcu et al. 2015; Dixon et al. 2015). To understand the link between compartment switching and gene expression upon SMARCA4 knockdown, we plotted the shSMARCA4/ shSCRAM $\log _{2}$ fold change RNA-seq expression levels of the genes that were located either within unchanged compartments or within regions that switched compartments (Fig. 5C). The genes located in regions with a compartment switch from A-type to B-type upon SMARCA4 knockdown showed significantly lower expression levels than the genes within unaltered compartment regions. The differences in gene expression within B-type to A-type compartmentswitching regions (shSMARCA4 to shSCRAM), although statistically significant (Wilcoxon rank-sum test; $P=0.02$ ), showed much more similar expression levels upon SMARCA4 knockdown (Fig. 5C). Nevertheless, these results suggest a prominent correlation between differential compartmentalization and gene expression.

Next, we asked whether the regions with SMARCA4 binding were associated with compartment switching. Of all SMARCA4bound sites, $76 \%$ were located within the constitutive open Atype compartments. In contrast, $21 \%$ of SMARCA4 ChIP-seq peaks were found in the closed B-type compartment regions (Fig. 5D), and $\sim 3 \%$ of SMARCA4 peaks were in regions showing compartment-switching (Fig. 5D). We then assessed the percentage of genomic compartment-switching regions that were either bound or unbound by SMARCA4. We observed that SMARCA4 was bound to $\sim 80 \%$ of constitutive A-type compartments (A to A) and $~ 50 \%$ of constitutive B-type compartments (B to B) (Fig. 5E). The frequency of SMARCA4 binding to altered compartment regions was similar, because $75 \%$ of "A to B" and $55 \%$ of "B to A" compartment-switching regions showed SMARCA4 binding, suggesting a similar degree of localization at compartment-switching regions. This suggests that SMARCA4 is therefore not likely to be directly mediating the compartment switch.

Taken together, these results show that SMARCA4 knockdown affects compartment organization in the genome. Although this change is confined to a subset of the genome, it is associated with differential gene expression.

\section{SMARCA4 is associated with TAD boundaries and TAD boundary strength}

Each compartment is composed of TADs, which are sub-megabasescale structures constituting a confined nuclear microenvironment for the proper association and regulation of promoters and enhancers (Nora et al. 2013). MCF-10A shSCRAM and shSMARCA4 interaction maps at 40-kb resolution revealed these 


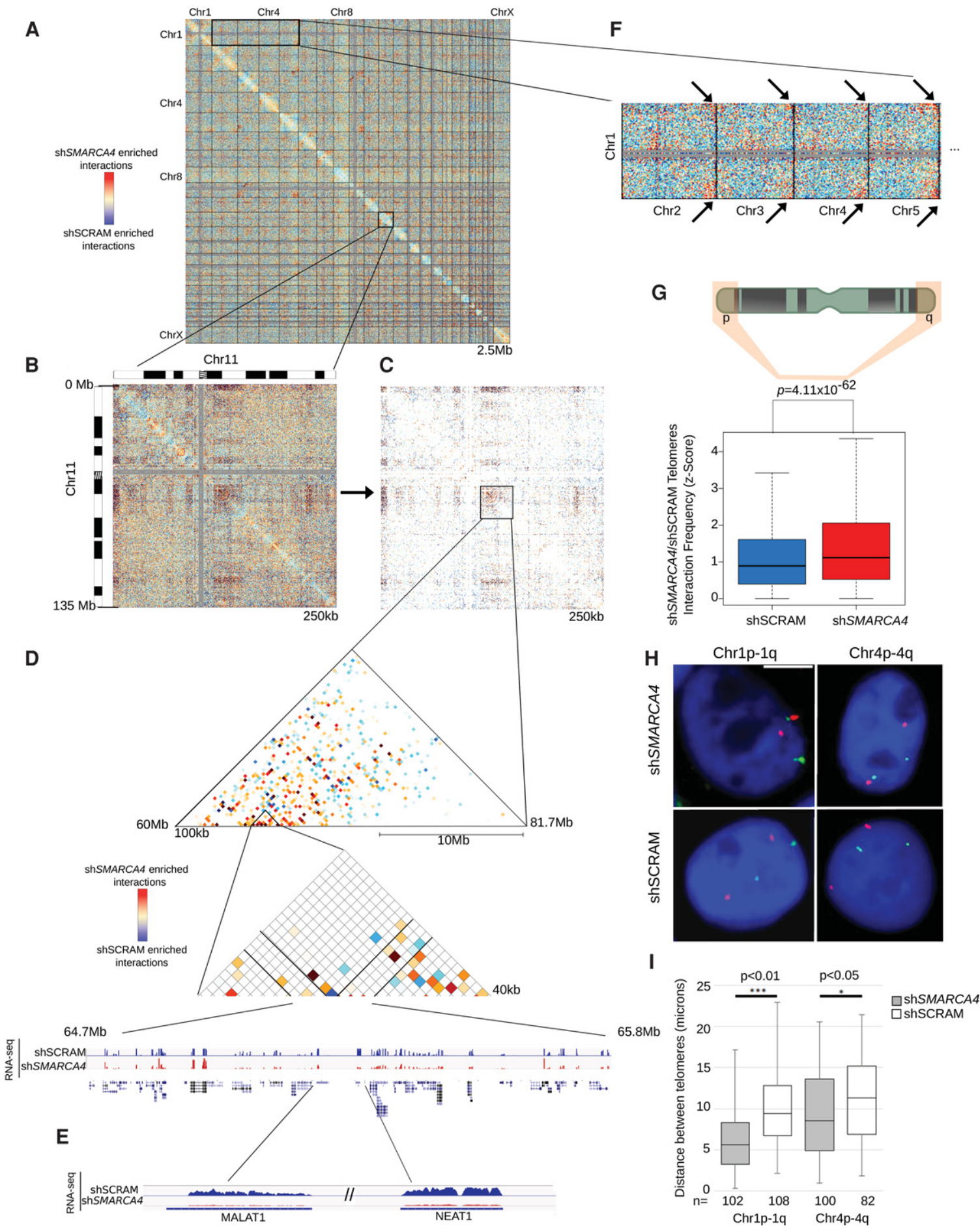

Figure 4. (A) Genome wide interaction heatmap at $2.5 \mathrm{Mb}$ resolution showing the differences between interactions that are gained and lost upon SMARCA4 knockdown. The chromosomes are stacked from top-left to bottom-right in order (Chr 1, Chr 2, .., Chr 22, and Chr X). (B) A zoom-in of Chr 11 at 250-kb resolution showing all differential interactions. (C) The interactions that are altered with significance (see Methods). (D) A further zoom-in view of a genomic region on Chr 11 (Chr 11: 60000001-81750000) (top) showing the differential interactions where the MALAT1 and NEAT1 loci reside (Chr 11: 64750339-65807685). (E) RNA-seq tracks from shSMARCA4 and shSCRAM cells showing a reduction of expression in NEAT1 and MALAT1 IncRNA genes upon SMARCA4 knockdown. (F) A zoom-in of the inter-chromosomal interactions between $\mathrm{Chr} 1$ and $\mathrm{Chr} 2$ through Chr 5, with arrows indicating the enriched telomeric interactions in the shSMARCA4 cells. This pattern of subtelomeric interaction occurs throughout the genome. (G) Quantification of the interactions among subtelomeric ends for shSCRAM and shSMARCA4 Hi-C data sets. The subtelomeric ends show significantly (Student's $t$-test: $P<0.01$ for $\mathrm{Chr} 1$ and $P<0.05$ for $\mathrm{Chr} 4$ ) higher frequency of interactions in shSMARCA4 cells compared to control cells. (H) DNA-FISH images of shSMARCA4 and shSCRAM cells showing the intra-chromosomal telomeric interactions of Chr 1 and Chr 4 . (I) Box plot showing the quantification of the telomere distances in shSMARCA4 and shSCRAM cells, quantified as described in the methods. P-value: Student's t-test. 
A
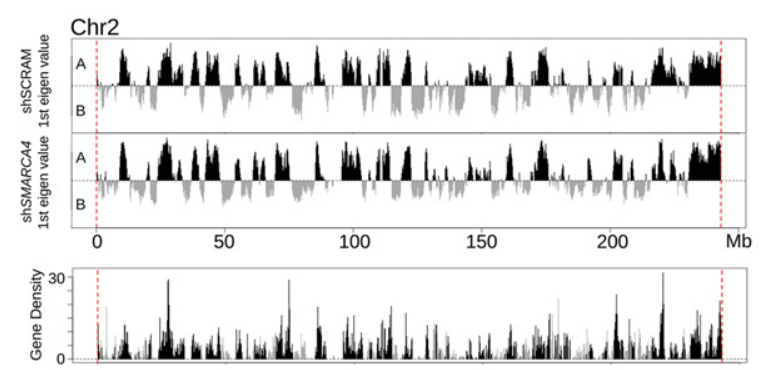

C

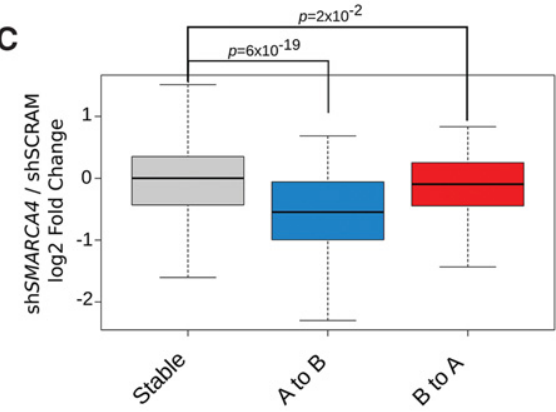

shSCRAM to shSMARCA4 Compartment Switch

E



B

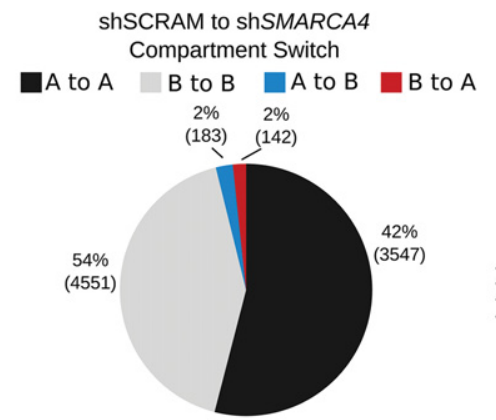

D
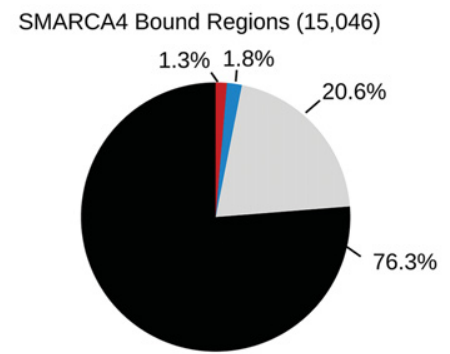

$\mathrm{A}$ to $\mathrm{A} \square \mathrm{B}$ to $\mathrm{B} \square \mathrm{A}$ to $\mathrm{B} \square \mathrm{B}$ to $\mathrm{A}$ shSCRAM to ShSMARCA4 Compartment Switch

Figure 5. (A) Compartment profiles (the first principal components) of shSCRAM and shSMARCA4 data for Chr 2. The A-type (open) compartments are shown in black, and the B-type (closed) compartments are shown in gray. The same color scheme was used for the gene density plot for Chr 2 in the lower panel. (B) Pie chart showing the genomic compartment changes between shSCRAM and shSMARCA4 data sets. " $A$ " and " $B$ " denote the open and closed compartments, respectively. "A to $A^{\prime \prime}$ represents compartments that are open in both cell lines; "B to $B$ " represents compartments that are closed in both cell lines; "A to B" denotes compartments that are open in shSCRAM but closed in shSMARCA4; and "B to A" denotes compartments that are closed in shSCRAM and open in shSMARCA4. (C) shSMARCA4/shSCRAM $\log _{2}$ fold change RNA-seq expression box plot of all the genes residing at regions for different compartmental switch categories. The compartments that are switched from A to B and from B to A show significantly decreased and increased expression levels, respectively. P-value: Wilcoxon rank-sum test. (D) Pie chart showing the compartment-switching profiles of SMARCA4-bound regions. $(E)$ Bar graph showing the percentage of the compartment-switching regions that are bound by SMARCA4. The colored portions of the graph denote the SMARCA4-bound percentage of each compartment-switching category.

sub-megabase-scale interaction domains on all chromosomes, suggesting that SMARCA4 knockdown does not result in a loss of TAD formation (Fig. 6A; Supplemental Fig. S8A,B). In order to quantify the TAD boundary scores and identify specific TADs, we generated insulation plots using a method that was described previously (Barutcu et al. 2015; Crane et al. 2015). We identified 2963 and 2796 TAD boundaries in shSCRAM and shSMARCA4 MCF-10A cells, respectively (Fig. 6B). The identified TADs showed known characteristics, such as increased density of genes and Pol II binding at the boundaries compared to the surrounding regions (Supplemental Fig. S8C,D). Despite the notion that TADs are stable across different cell types, species, and different biological contexts (Dixon et al. 2012, 2015; Nora et al. 2012, 2013), interestingly, SMARCA4 knockdown altered the localization of $\sim 14 \%$ of the
TAD boundaries. The majority of TAD boundaries $(83 \%$ of shSCRAM and $88 \%$ of shSMARCA4) were overlapping between the control and the SMARCA4 knockdown cells (Fig. 6B).

TAD boundaries are bound by proteins such as CTCF and cohesin in vertebrates (Vietri Rudan et al. 2015) and by several architectural binding proteins in flies (Van Bortle et al. 2014). Therefore, we asked whether SMARCA4 plays any role in TAD boundaries and assessed whether SMARCA4 localization was enriched at TAD boundaries. Intersection of SMARCA4 ChIP-seq peaks with both the shSMARCA4 and shSCRAM TAD boundary definitions yielded similar results, in which $\sim 25 \%$ of all SMARCA4 binding was located at TAD boundaries (Fig. 6C). Surprisingly, we observed an enrichment of SMARCA4 binding at the boundaries when the frequency of SMARCA4 binding was 
A

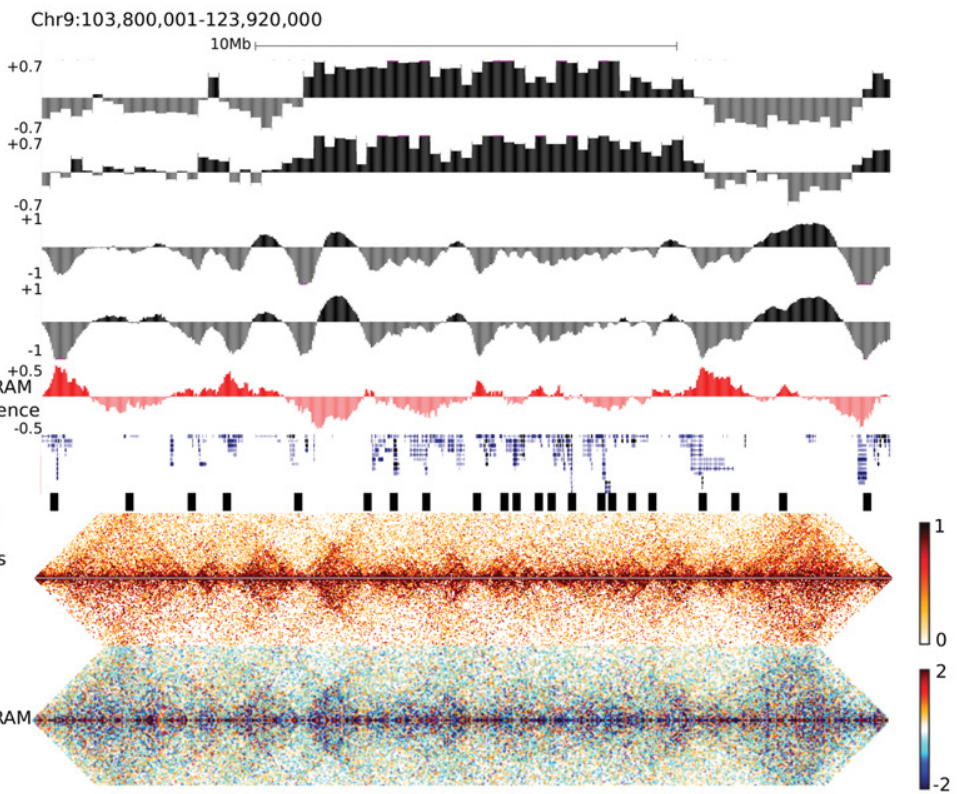

B

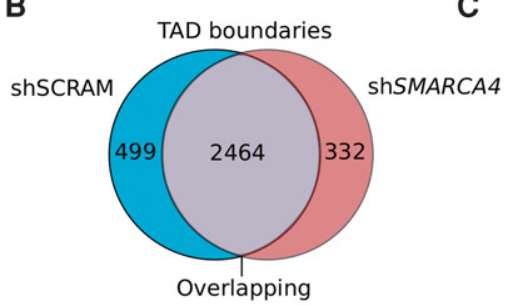

E

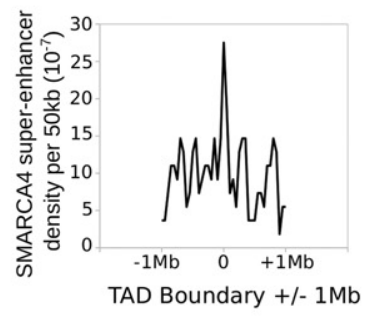

C

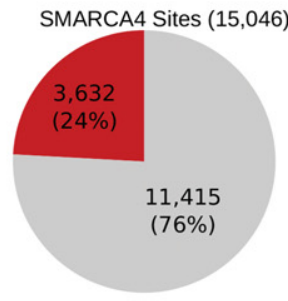

At TAD Boundaries Not at TAD Boundaries

F

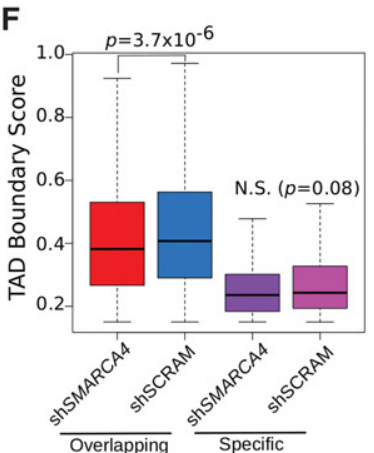

D

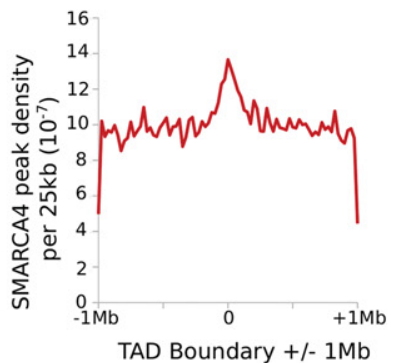

G

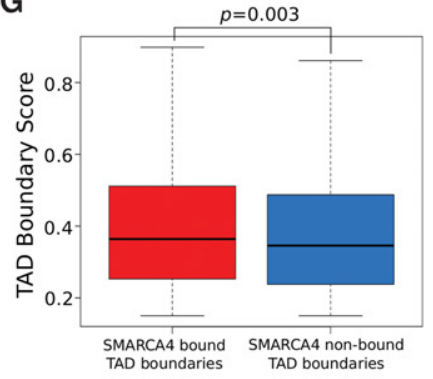

Figure 6. (A) An example of a region on Chr 9 (Chr 9: 103800001-123920000) showing (from top to bottom) the compartment profiles of shSMARCA4 and shSCRAM at 250-kb intervals, the insulation plot profiles at 40-kb intervals (see Methods), the insulation plot difference between shSMARCA4 and shSCRAM, hg19 UCSC genes, TAD boundaries, shSMARCA4 and shSCRAM contact heatmaps showing the TADs, and a subtraction of the shSCRAM from the shSMARCA4 contact heatmap. (B) Venn diagram showing that the TAD boundaries are largely similar between shSCRAM and shSMARCA4 Hi$C$ data sets. (C) Pie chart showing the percentage of SMARCA4 localization at TAD boundaries. $(D)$ The frequency plot of SMARCA4 ChIP-seq peaks per $25 \mathrm{~kb}$ for $\pm 1 \mathrm{Mb}$ of every shSMARCA4 TAD boundary. (E) The frequency plot of SMARCA4 super-enhancers per $50 \mathrm{~kb}$ for $\pm 1 \mathrm{Mb}$ of every shSMARCA4 TAD boundary. (F) Box plot showing the TAD boundary score distribution for the overlapping and the shSCRAM- and shSMARCA4-specific TAD boundaries. $P$-value: Wilcoxon rank-sum test. $(G)$ SMARCA4 binding is associated with higher (Wilcoxon rank-sum test; $P=0.003$ ) TAD boundary score. Box plot showing the TAD boundary scores for SMARCA4-bound and unbound TAD boundaries.

plotted around the TAD borders (Fig. 6D). Sixty-six percent of all TAD boundaries were bound by SMARCA4. A similar phenomenon was observed when SMARCA4-bound super-enhancers were plotted across the TAD boundaries (Fig. 6E).
The strength of a TAD boundary is a measure of the allowance of inter-TAD interactions across the boundary (Van Bortle et al. 2014; Li et al. 2015). Although our data indicate that the majority of TAD boundaries are similar, we wondered whether SMARCA4

\section{Genome Research}

www.genome.org 
knockdown resulted in a change in TAD boundary strength. Interestingly, SMARCA4 knockdown resulted in an overall decrease (Wilcoxon rank-sum test; $P=3.7 \times 10^{6}$ ) in the overlapping TAD boundary strength, as shown by plotting the boundary scores for the overlapping and unique TAD boundaries for the shSMARCA4 and shSCRAM cells (Fig. 6F). Furthermore, to address whether this decrease is related to SMARCA4 binding, we compared the scores of the TAD boundaries that were either SMARCA4-bound or not. We observed a significant decrease (Wilcoxon rank-sum test; $P=0.003$ ) of TAD boundary strength at borders that lacked SMARCA4 binding (Fig. 6G).

CTCF is an important component of TAD boundaries. When we intersected a previously published MCF-10A CTCF ChIP-seq data set (Ross-Innes et al. 2011) with SMARCA4 peaks, 10\% of all SMARCA4 peaks and $12 \%$ of the SMARCA4 peaks that are located on a TAD boundary directly overlapped with CTCF, implying a crosstalk between SMARCA4 and CTCF at least for a subset of bound genomic regions (Supplemental Fig. S8E,F). The intersection of these two factors increased by twofold when the vicinity $( \pm 1 \mathrm{~kb})$ of both SMARCA4 and CTCF peaks were considered. To assess whether the effects of SMARCA4 on TAD boundaries result from changes in the local chromatin structure around the CTCF sites, we analyzed publicly available SMARCA4-dependent accessibility and nucleosome positioning DNase I- and MNase-seq data sets (Tolstorukov et al. 2013; Morris et al. 2014; Stavreva et al. 2015). SMARCA4 perturbation affected nucleosome positioning within hundreds of base pairs of CTCF binding sites in mouse fibroblast cells (Supplemental Fig. S9A-C), showing decreased nucleosome occupancy immediately around the CTCF binding sites. SMARCA4 perturbation also altered chromatin accessibility in murine epithelial cells (Supplemental Fig. S9D). Taken together, these results demonstrate a role for SMARCA4, likely via mediating the local chromatin accessibility around the CTCF sites.

\section{Discussion}

Modification of the chromatin structure by ATP-dependent remodeling complexes is an essential process in transcriptional regulation. SMARCA4 affects both the activation and repression of many genes through its interactions with transcription factors and other cofactors (Trotter and Archer 2008). To understand the role of SMARCA4 in genome architecture, we characterized the SMARCA4-dependent alterations in gene expression and higherorder chromatin structure in mammary epithelial MCF-10A cells. RNA-seq analysis in the control and SMARCA4 knockdown cells showed an extensive down-regulation of genes related to ECM components and up-regulation of genes related to lipid metabolism and calcium signaling (Fig. 1). SMARCA4 is linked to calcium signaling (Lai et al. 2009; Nasipak et al. 2015). Literature also supports the idea that SMARCA4 (or the mammalian SWI/SNF complex) regulates lipid metabolism, as it was shown that SMARCA4 regulates PPARG expression, which in turn regulates lipid metabolism in differentiating adipocytes (Salma et al. 2004). In addition, SMARCD3, a subunit of the mammalian SWI/SNF complex, is required for activation of fatty acid and triglyceride synthesis in response to insulin and to feeding in liver (Wang et al. 2013). Interestingly, SMARCA4 binding was enriched at down-regulated genes. The up-regulation of genes could be an indirect effect of SMARCA4 knockdown, because in many cases, these genes were not directly bound by SMARCA4 (Fig. 2E).

Growing evidence suggests that the shape of the nucleus, the stiffest organelle in the cell, might partly be affected by force-in- duced changes, a phenomenon known as nuclear mechanotransduction (Dahl et al. 2008). It is becoming well established that the cell surface adhesion receptors, such as integrins and cadherins, can exert mechanical forces to the nucleus and can potentially cause gene activation and/or chromatin reorganization (Wang et al. 2009). However, changes in nuclear shape can be induced from either external forces exerted by the cytoskeleton or via internal nuclear forces. Previous work showed that SMARCA4 knockdown results in nuclear shape alterations in MCF-10A cells (Imbalzano et al. 2013b). However, the disruption of the cytoplasmic filaments (actin, tubulin, and cytokeratins) did not alter SMARCA4-dependent structural changes observed in the MCF10A cells (Imbalzano et al. 2013b). This implies that SMARCA4, apart from its chromatin remodeling function, might have additional roles in maintaining the structural integrity of the nucleus (Imbalzano et al. 2013a). Interestingly, in the present study, we determined that many of the genes that are down-regulated following SMARCA4 knockdown were associated with the extracellular matrix. These findings suggest an additional mechanism for SMARCA4-mediated regulation of nuclear integrity via regulation of ECM genes and possible alteration of cell surface connections and mechanotransducing forces to the nucleus.

Hi-C analysis of SMARCA4 knockdown and control MCF-10A cells revealed a significant enrichment of subtelomeric interactions in the shSMARCA4 cells (Fig. 4F-I). Literature evidence connecting SWI/SNF enzymes and telomeres is limited. SMARCA4 knockdown was shown to result in increased telomerase expression that leads to increased telomere lengths (Wu et al. 2014). Yeast SWI/SNF is required for telomeric silencing (Dror and Winston 2004), and telomeric expansion (Tomar et al. 2008). Mammalian cells lacking SMARCAL1, which is a SNF2 family ATPase but is not a subunit of the mammalian SWI/SNF complex, accumulate elevated levels of telomere-associated DNA damage (Poole et al. 2015). Moreover, knockdown of the mammalian SWI/SNF enzyme subunit, ARID1A, reduces telomeric repeatcontaining RNA (TERRA) levels by at least twofold (Scheibe et al. 2013). Further experiments assessing whether SMARCA4 affects nucleosome positioning at or enhances/interferes with binding of specific proteins at telomeres to affect higher-order telomeric structures will shed further light on the relationship between SMARCA4 and telomere organization. Recently, Guidi et al. (2015) showed that the telomeres of yeast cells undergo spatial reorganization upon switching to different metabolic states. Because our RNA-seq analysis showed up-regulation of genes related to lipid synthesis and since our Hi-C analysis showed alterations in telomeric interactions in shSMARCA4 cells, it is tempting to speculate that the increased telomeric interactions (Fig. 4F-I) may be associated with changes in the metabolic state of the shSMARCA4 cells.

The importance of nuclear structure-gene expression relationships is supported by our finding that $4 \%$ of genomic compartments are altered in a SMARCA4-dependent manner (Fig. 4B). The magnitude of the functional impact of SMARCA4 on the architectural landscape is illustrated by previous reports showing that comparisons of relative compartmental differences in distinct cell types ranged from $4 \%$ to $25 \%$ of the genome (Barutcu et al. 2015; Dixon et al. 2015). The compartmental changes are associated with gene expression (Fig. 5C). However, it is still debated whether compartmental change or chromosomal movement causes differential gene expression or vice versa. There is evidence supporting both viewpoints (Chuang and Belmont 2007; Therizols et al. 2014). 
The formation of topologically associating domain boundaries is dependent on many factors (Van Bortle et al. 2014; CubeñasPotts and Corces 2015), especially insulators such as CTCF and cohesin. Here, we report the rather remarkable observation that SMARCA4 peaks and super-enhancers were also enriched at TAD boundaries (Fig. 6D,E), and the depletion of SMARCA4 resulted in $\sim 14 \%$ of TAD boundaries being altered and lower TAD boundary scores genome-wide (Fig. 6F). Furthermore, SMARCA4-bound boundaries exhibited a stronger boundary score than the boundaries not bound by SMARCA4 (Fig. 6G). Therefore, in addition to well-studied insulators such as CTCF and cohesin, maintenance of TAD boundaries is affected, directly or indirectly, by SMARCA4, and by extension, the mammalian SWI/SNF enzyme possibly via regulating CTCF and cohesin binding. A previous report showed that SMARCA4 and other subunits of the mammalian SWI/SNF complex bind near regions critical for genome organization (e.g., CTCF and lamin binding sites and DNA replication origins) (Euskirchen et al. 2011). Our analysis of publicly available (Tolstorukov et al. 2013; Morris et al. 2014) DNase I accessibility and nucleosome positioning data around the CTCF sites demonstrated a SMARCA4-dependent effect on local chromatin structure (Supplemental Fig. S9), similar to the effect of SMARCA4 knockdown that was previously noted around TSS of known genes (Tolstorukov et al. 2013). Further experiments investigating whether SMARCA4 is required for CTCF, as well as cohesin and mediator binding, would provide insight into the mechanism by which SMARCA4 affects TAD boundaries.

Despite affecting genome organization at multiple levels, the overall genome organization was largely preserved upon SMARCA4 knockdown. Recent studies knocking down other fundamental chromatin organizers, CTCF (Zuin et al. 2014), cohesin (Seitan et al. 2013), and histone H1 (Geeven et al. 2015) similarly showed little effect on overall genome organization as assayed by Hi-C. It is possible that the remaining SMARCA4 levels in the knockdown MCF-10A cells (Fig. 1A) resulted in more modest changes than otherwise might have been expected. It is also likely that there are redundant molecular mechanisms in the cell to ensure the integrity of the genome and the nucleus. In the shSMARCA4 MCF-10A cells, the SMARCA4 homolog SMARCA2 (also known as BRM) still exists, and we previously reported that MCF-10A cells are not viable when both SMARCA4 and SMARCA2 are simultaneously knocked down (Cohet et al. 2010). Total removal of SMARCA4 by CRISPR/Cas9 in the MDA-MB-231 human metastatic breast cancer cell line and genetic ablation in primary myoblasts also resulted in cell death (Padilla-Benavides et al. 2015; Wu et al. 2015). These findings suggest that the importance of SMARCA4 in cell viability may preclude observing more severe effects on genome organization.

Taken together, we identify novel roles for SMARCA4 in regulating higher-order chromatin structure by affecting telomere organization, TAD boundary strength, and the frequency and specificity of long-range chromatin interactions.

\section{Methods}

\section{Cell culture}

MCF-10A cells expressing control shRNA and shRNA targeting SMARCA4 were generated as previously described (Cohet et al. 2010). The cells were maintained in monolayer in Dulbecco's modified Eagle's medium-F12 (DMEM/F12) (Invitrogen, 21041025) supplemented with 5\% horse serum (Invitrogen,
16050122), 1\% penicillin/streptomycin (Invitrogen, 15140122), $0.5 \mu \mathrm{g} / \mathrm{mL}$ hydrocortisone (Sigma, H-0888), $100 \mathrm{ng} / \mathrm{mL}$ cholera toxin (Sigma, C-8052), $10 \mu \mathrm{g} / \mathrm{mL}$ insulin (Sigma, I-1882), and 20 $\mathrm{ng} / \mathrm{mL}$ recombinant human EGF (Peprotech, 100-15) as described previously (Debnath et al. 2003). The doxycyline induction was performed by the addition of $0.05 \mu \mathrm{g} / \mathrm{mL}$ doxycycline to the cells and incubating them for 3-4 d.

\section{RNA-seq and analysis}

RNA was isolated from MCF-10A cells at $~ 75 \%$ confluence using the TRIzol Reagent (Life Technologies \#15596-026), including treatment with DNase I. The poly(A)-selected RNA-seq libraries were generated with TruSeq RNA Sample Preparation Kit v2, and single-end 100-bp sequencing was performed using a HiSeq 2000 instrument. RNA-seq analysis was performed by filtering and mapping the reads by Bowtie 2 (Langmead and Salzberg 2012), quantifying the transcripts by RSEM v1.2.7 (Li and Dewey 2011), and finding the differentially expressed genes $\left(\log _{2}\right.$ fold change $>1, P$ $<0.01$ ) by DESeq2 (Love et al. 2014).

\section{Preparation of Hi-C libraries}

Hi-C was performed as previously described with minor modifications (Belton et al. 2012). The modification was in the biotin incorporation step, in which the mixture was incubated for $40 \mathrm{~min}$ at $37^{\circ} \mathrm{C}$. The MCF-10A shSCRAM and shSMARCA4 samples displayed a range of $25 \%-50 \%$ biotin incorporation efficiency. At the end of $\mathrm{Hi}-\mathrm{C}$ sample preparation, the libraries were sequenced using paired-end 100-bp reads with a HiSeq 2000 instrument.

\section{Read mapping/binning/ICE correction}

Supplemental Table S3 summarizes the mapping results and different classes of reads and interactions observed (Lajoie et al. 2015). The data were binned at $2.5 \mathrm{Mb}, 1 \mathrm{Mb}, 250 \mathrm{~kb}, 100 \mathrm{~kb}$, and $40 \mathrm{~kb}$ nonoverlapping genomic intervals. In our Hi-C analyses, we utilized the iterative correction and eigenvector decomposition (ICE) method (Imakaev et al. 2012). The replicates showed high correlation (Pearson correlation; $R^{2}$ ranging from 0.69 to 0.92 ) at multiple scales (Supplemental Fig. S4). For the downstream analyses, sequences obtained from both biological replicates were pooled and ICE-corrected to serve as a combined data set.

\section{Z-score calculation}

We calculated the $Z$-scores by modeling the overall Hi-C decay with distance using a modified LOWESS method ( $\alpha=1 \%$, IQR filter), as described previously (Sanyal et al. 2012). LOWESS calculates the weighted-average and weighted-standard deviation for every genomic distance and therefore normalizes for genomic distance signal bias.

\section{Calculation of differential interactions}

To capture the differences between shSCRAM and shSMARCA4 interactions, we used a method previously described (Barutcu et al. 2015). Briefly, we first transformed the Hi-C data into $Z$-score matrices for all four replicate data sets (shSMARCA4-R1, shSMARCA4R2, shSCRAM-R1, and shSCRAM-R2). For each interaction, the mean sample:sample (between samples) $Z$-score difference was calculated from all pairwise combinations of the four data sets (shSMARCA4-R1-shSCRAM-R1， shSMARCA4-R1-shSCRAMR2, shSMARCA4-R2-shSCRAM-R1， shSMARCA4-R2-shSCRAM$\mathrm{R} 2$ ). The replicate:replicate $Z$-score difference (within samples) was also calculated for a random set of 500,000 interactions. These random replicate:replicate $Z$-score differences were then

\section{Genome Research}

www.genome.org 
used to build an expected distribution of $Z$-score differences. The resulting $Z$-score difference matrix was then derived by calculating for each bin the ratio of the mean of the set of four possible sample:sample $Z$-score differences minus the genome-wide mean of the replicate:replicate $Z$-score difference, divided by the genome-wide standard error of the replicate:replicate $Z$-score differences. All statistical analyses have been corrected for multiple correction tests.

\section{Compartment profiles}

To detect the genomic compartments, first, Pearson correlation of the $Z$-score matrices was calculated. In performing principal component analysis (Lieberman-Aiden et al. 2009; Zhang et al. 2012), the first principal component detects the patterns of increased and decreased interaction across the genome that appear as a plaid pattern in the heatmap. Each genomic region matches this prominent interaction pattern (positive eigenvector value) or its opposite (negative eigenvector value), and these represent the two spatially segregated compartments. The open, gene rich "A-type" compartment may end up with either a positive or negative eigenvector. To detect which compartment is the open "A-type" and which is the closed "B-type," the genome-wide gene density was calculated to assign the "A-type" and "B-type" compartmentalization.

\section{Identification of TAD boundaries (insulation square analysis)}

TAD calling was performed as calculating the insulation score of each bin using the 40-kb resolution combined Hi-C data as previously described (Barutcu et al. 2015; Crane et al. 2015).

\section{ChIP-seq analysis}

The ChIP assay was performed as previously described (Lee et al. 2006). The chromatin was sheared by using a Bioruptor instrument on high setting, $30 \mathrm{sec}$ on and $30 \mathrm{sec}$ off, for 5 min for five cycles. The pull-down was performed using a SMARCA4 antibody (Santa Cruz \#G-7). The pull-down and input control sequencing libraries were generated using the NEXTflex Rapid DNA Sequencing Kit (Bioo Scientific \#5144-02) and were sequenced by using singleend 100-bp reads with a HiSeq 2000 instrument. The adapters were trimmed from the sequencing reads, and the reads were aligned to the hg19 human genome using the Bowtie 2 tool (Langmead and Salzberg 2012). Quality controls, peak calling, motif analysis and peak annotation were performed using the HOMER suite (Heinz et al. 2010). As the ChIP signal across the biological replicates showed high correlation (Pearson correlation;, $R^{2}=0.72$ ) (Supplemental Fig. S3B), we performed ChIP-seq peak calling on the pooled replicates.

\section{DNA fluorescence in situ hybridization (FISH)}

FISH was performed as described previously (Sehgal et al. 2016) on MCF-10A cells induced to express either shSCRAM or shSMARCA4 shRNAs. Probes from bacterial artificial chromosomes RP1182D16 for Chr 1p, RP11-81J5 for Chr 1q, RP11-81L5 for Chr 4p; and RP11-196K19 for Chr 4q were used. shRNA expression was confirmed via GFP expression and by immunofluorescence for SMARCA4. Cell images were acquired using an epifluorescence Zeiss AxioImager microscope equipped with a Hamamatsu charged coupled device (CCD) camera in a series of stacked images (n, shSCRAM Chr 1, 108; shSMARCA4 Chr 1, 102; shSCRAM Chr 4, 82; shSMARCA4 Chr 4, 100). Images were captured using $100 \times$ objective magnification and Zen 2011 imaging software (Zeiss). Image J's coordinate function was used to identify $3 \mathrm{D}$ coordinates of probes and subsequently run through the eFISHent program for distance measurements (Fritz et al. 2014).

\section{Data access}

The raw and processed RNA-seq, ChIP-seq, and Hi-C data sets from this study have been submitted to the NCBI Gene Expression Omnibus (GEO; http://www.ncbi.nlm.nih.gov/geo/) under accession number GSE74716.

\section{Acknowledgments}

We thank Imbalzano and Stein laboratory members for critical discussion; Alper Kucukural for technical help with RNA-seq analysis; Seda Barutcu for critical reading of the manuscript and scientific input; Scott Tighe, Robert Devins, and Jonathan Gordon for technical help with deep-sequencing; and Tara Smith for technical assistance. The next-generation sequencing was performed in the University of Vermont Advanced Genome Technologies Core Massively Parallel Sequencing Facility, which was supported by the University of Vermont Cancer Center, Lake Champlain Cancer Research Organization, UVM College of Agriculture and Life Sciences, and the UVM College of Medicine. This work was supported by National Institutes of Health (NIH) grants P01 CA082834 and R01 HG003143. J.D. is an investigator of the Howard Hughes Medical Institute.

Author contributions: A.R.B., G.S.S., A.N.I., J.L.S., A.J.v.W., and J.B.L. conceived the project; A.R.B. performed and analyzed the RNA-seq and ChIP-seq experiments and performed the Hi-C experiments; B.R.L., R.P.M., and J.D. performed the initial Hi-C analysis; A.R.B. performed the secondary Hi-C bioinformatic analyses with input from J.D., B.R.L., and R.P.M.; A.J.F. and J.A.N. designed FISH experiments; and A.J.F. performed and analyzed FISH experiments. All authors discussed the results, and A.R.B. wrote the manuscript with input from all the authors. All authors have read and approved the manuscript.

\section{References}

Barutcu AR, Lajoie BR, McCord RP, Tye CE, Hong D, Messier TL, Browne G, van Wijnen AJ, Lian JB, Stein JL, et al. 2015. Chromatin interaction analysis reveals changes in small chromosome and telomere clustering between epithelial and breast cancer cells. Genome Biol 16: 214.

Belton JM, McCord RP, Gibcus JH, Naumova N, Zhan Y, Dekker J. 2012. Hi$\mathrm{C}$ : a comprehensive technique to capture the conformation of genomes. Methods 58: 268-276.

Bossen C, Murre CS, Chang AN, Mansson R, Rodewald HR, Murre C. 2015. The chromatin remodeler Brg1 activates enhancer repertoires to establish B cell identity and modulate cell growth. Nat Immunol 16: 775-784.

Bultman S, Gebuhr T, Yee D, La Mantia C, Nicholson J, Gilliam A, Randazzo F, Metzger D, Chambon P, Crabtree G, et al. 2000. A Brg1 null mutation in the mouse reveals functional differences among mammalian SWI/ SNF complexes. Mol Cell 6: 1287-1295.

Bultman SJ, Herschkowitz JI, Godfrey V, Gebuhr TC, Yaniv M, Perou CM, Magnuson T. 2008. Characterization of mammary tumors from Brg1 heterozygous mice. Oncogene 27: 460-468.

Chuang CH, Belmont AS. 2007. Moving chromatin within the interphase nucleus-controlled transitions? Semin Cell Dev Biol 18: 698-706.

Clapier CR, Cairns BR. 2009. The biology of chromatin remodeling complexes. Annu Rev Biochem 78: 273-304.

Cohet N, Stewart KM, Mudhasani R, Asirvatham AJ, Mallappa C, Imbalzano KM, Weaver VM, Imbalzano AN, Nickerson JA. 2010. SWI/SNF chromatin remodeling enzyme ATPases promote cell proliferation in normal mammary epithelial cells. J Cell Physiol 223: 667-678.

Crane E, Bian Q, McCord RP, Lajoie BR, Wheeler BS, Ralston EJ, Uzawa S, Dekker J, Meyer BJ. 2015. Condensin-driven remodelling of X chromosome topology during dosage compensation. Nature 523: 240-244.

Cremer T, Cremer M, Dietzel S, Muller S, Solovei I, Fakan S. 2006. Chromosome territories-a functional nuclear landscape. Curr Opin Cell Biol 18: 307-316. 
Croft D, Mundo AF, Haw R, Milacic M, Weiser J, Wu G, Caudy M, Garapati P, Gillespie M, Kamdar MR, et al. 2014. The Reactome pathway knowledgebase. Nucleic Acids Res 42(Database issue): D472-D477.

Cubeñas-Potts C, Corces VG. 2015. Architectural proteins, transcription, and the three-dimensional organization of the genome. FEBS Lett $\mathbf{5 8 9}$ (20 Pt A): 2923-2930.

Cutter AR, Hayes JJ. 2015. A brief review of nucleosome structure. FEBS Lett 589(20 Pt A): 2914-2922.

Dahl KN, Ribeiro AJ, Lammerding J. 2008. Nuclear shape, mechanics, and mechanotransduction. Circ Res 102: 1307-1318.

Debnath J, Muthuswamy SK, Brugge JS. 2003. Morphogenesis and oncogenesis of MCF-10A mammary epithelial acini grown in three-dimensional basement membrane cultures. Methods 30: 256-268.

Dixon JR, Selvaraj S, Yue F, Kim A, Li Y, Shen Y, Hu M, Liu JS, Ren B. 2012. Topological domains in mammalian genomes identified by analysis of chromatin interactions. Nature 485: 376-380.

Dixon JR, Jung I, Selvaraj S, Shen Y, Antosiewicz-Bourget JE, Lee AY, Ye Z, Kim A, Rajagopal N, Xie W, et al. 2015. Chromatin architecture reorganization during stem cell differentiation. Nature 518: 331-336.

Dror V, Winston F. 2004. The Swi/Snf chromatin remodeling complex is required for ribosomal DNA and telomeric silencing in Saccharomyces cerevisiae. Mol Cell Biol 24: 8227-8235.

The ENCODE Project Consortium. 2012. An integrated encyclopedia of DNA elements in the human genome. Nature 489: 57-74.

Euskirchen GM, Auerbach RK, Davidov E, Gianoulis TA, Zhong G, Rozowsky J, Bhardwaj N, Gerstein MB, Snyder M. 2011. Diverse roles and interactions of the SWI/SNF chromatin remodeling complex revealed using global approaches. PLoS Genet 7: e1002008.

Flaus A, Owen-Hughes T. 2011. Mechanisms for ATP-dependent chromatin remodelling: the means to the end. FEBS J 278: 3579-3595.

Fritz AJ, Stojkovic B, Ding H, Xu J, Bhattacharya S, Berezney R. 2014. Cell type specific alterations in interchromosomal networks across the cell cycle. PLoS Comput Biol 10: e1003857.

Geeven G, Zhu Y, Kim BJ, Bartholdy BA, Yang SM, Macfarlan TS, Gifford WD, Pfaff SL, Verstegen MJ, Pinto H, et al. 2015. Local compartment changes and regulatory landscape alterations in histone H1-depleted cells. Genome Biol 16: 289 .

Guidi M, Ruault M, Marbouty M, Loïodice I, Cournac A, Billaudeau C, Hocher A, Mozziconacci J, Koszul R, Taddei A. 2015. Spatial reorganization of telomeres in long-lived quiescent cells. Genome Biol 16: 206.

Harada A, Mallappa C, Okada S, Butler JT, Baker SP, Lawrence JB, Ohkawa Y, Imbalzano AN. 2015. Spatial re-organization of myogenic regulatory sequences temporally controls gene expression. Nucleic Acids Res 43: 2008-2021.

Heinz S, Benner C, Spann N, Bertolino E, Lin YC, Laslo P, Cheng JX, Murre C, Singh H, Glass CK. 2010. Simple combinations of lineage-determining transcription factors prime cis-regulatory elements required for macrophage and B cell identities. Mol Cell 38: 576-589.

Hill DA, Chiosea S, Jamaluddin S, Roy K, Fischer AH, Boyd DD, Nickerson JA, Imbalzano AN. 2004. Inducible changes in cell size and attachment area due to expression of a mutant SWI/SNF chromatin remodeling enzyme. J Cell Sci 117(Pt 24): 5847-5854.

Ho L, Jothi R, Ronan JL, Cui K, Zhao K, Crabtree GR. 2009. An embryonic stem cell chromatin remodeling complex, esBAF, is an essential component of the core pluripotency transcriptional network. Proc Natl Acad Sci 106: $5187-5191$.

Hu G, Schones DE, Cui K, Ybarra R, Northrup D, Tang Q, Gattinoni L, Restifo NP, Huang S, Zhao K. 2011. Regulation of nucleosome landscape and transcription factor targeting at tissue-specific enhancers by BRG1. Genome Res 21: 1650-1658.

Imakaev M, Fudenberg G, McCord RP, Naumova N, Goloborodko A, Lajoie BR, Dekker J, Mirny LA. 2012. Iterative correction of Hi-C data reveals hallmarks of chromosome organization. Nat Methods 9: 999-1003.

Imbalzano AN, Imbalzano KM, Nickerson JA. 2013a. BRG1, a SWI/SNF chromatin remodeling enzyme ATPase, is required for maintenance of nuclear shape and integrity. Commun Integr Biol 6: e25153.

Imbalzano KM, Cohet N, Wu Q, Underwood JM, Imbalzano AN, Nickerson JA. 2013b. Nuclear shape changes are induced by knockdown of the SWI/SNF ATPase BRG1 and are independent of cytoskeletal connections. PLoS One 8: e55628.

Kadoch C, Hargreaves DC, Hodges C, Elias L, Ho L, Ranish J, Crabtree GR. 2013. Proteomic and bioinformatic analysis of mammalian SWI/SNF complexes identifies extensive roles in human malignancy. Nat Genet 45: $592-601$.

Kawaguchi T, Tanigawa A, Naganuma T, Ohkawa Y, Souquere S, Pierron G, Hirose T. 2015. SWI/SNF chromatin-remodeling complexes function in noncoding RNA-dependent assembly of nuclear bodies. Proc Natl Acad Sci 112: 4304-4309.

Kim SI, Bresnick EH, Bultman SJ. 2009a. BRG1 directly regulates nucleosome structure and chromatin looping of the $\alpha$ globin locus to activate transcription. Nucleic Acids Res 37: 6019-6027.
Kim SI, Bultman SJ, Kiefer CM, Dean A, Bresnick EH. 2009b. BRG1 requirement for long-range interaction of a locus control region with a downstream promoter. Proc Natl Acad Sci 106: 2259-2264.

King HA, Trotter KW, Archer TK. 2012. Chromatin remodeling during glucocorticoid receptor regulated transactivation. Biochim Biophys Acta 1819: $716-726$

Lai D, Wan M, Wu J, Preston-Hurlburt P, Kushwaha R, Grundström T, Imbalzano AN, Chi T. 2009. Induction of TLR4-target genes entails calcium/calmodulin-dependent regulation of chromatin remodeling. Proc Natl Acad Sci 106: 1169-1174.

Lajoie BR, Dekker J, Kaplan N. 2015. The Hitchhiker's guide to Hi-C analysis: practical guidelines. Methods 72: 65-75.

Lan X, Witt H, Katsumura K, Ye Z, Wang Q Bresnick EH, Farnham PJ, Jin VX. 2012. Integration of Hi-C and ChIP-seq data reveals distinct types of chromatin linkages. Nucleic Acids Res 40: 7690-7704.

Langmead B, Salzberg SL. 2012. Fast gapped-read alignment with Bowtie 2. Nat Methods 9: 357-359.

Lee TI, Johnstone SE, Young RA. 2006. Chromatin immunoprecipitation and microarray-based analysis of protein location. Nat Protoc 1: 729-748.

Li B, Dewey CN. 2011. RSEM: accurate transcript quantification from RNASeq data with or without a reference genome. BMC Bioinformatics 12: 323.

Li L, Lyu X, Hou C, Takenaka N, Nguyen HQ, Ong CT, Cubeñas-Potts C, Hu $\mathrm{M}$, Lei EP, Bosco G, et al. 2015. Widespread rearrangement of 3D chromatin organization underlies polycomb-mediated stress-induced silencing. Mol Cell 58: 216-231.

Lieberman-Aiden E, van Berkum NL, Williams L, Imakaev M, Ragoczy T, Telling A, Amit I, Lajoie BR, Sabo PJ, Dorschner MO, et al. 2009. Comprehensive mapping of long-range interactions reveals folding principles of the human genome. Science 326: 289-293.

Love MI, Huber W, Anders S. 2014. Moderated estimation of fold change and dispersion for RNA-seq data with DESeq2. Genome Biol 15: 550.

Marella NV, Malyavantham KS, Wang J, Matsui S, Liang P, Berezney R. 2009. Cytogenetic and CDNA microarray expression analysis of MCF10 human breast cancer progression cell lines. Cancer Res 69: 5946-5953.

Milacic M, Haw R, Rothfels K, Wu G, Croft D, Hermjakob H, D'Eustachio P, Stein L. 2012. Annotating cancer variants and anti-cancer therapeutics in reactome. Cancers 4: 1180-1211.

Morris SA, Baek S, Sung MH, John S, Wiench M, Johnson TA, Schiltz RL, Hager GL. 2014. Overlapping chromatin-remodeling systems collaborate genome wide at dynamic chromatin transitions. Nat Struct Mol Biol 21: 73-81.

Muchardt C, Yaniv M. 1993. A human homologue of Saccharomyces cerevisiae SNF2/SWI2 and Drosophila brm genes potentiates transcriptional activation by the glucocorticoid receptor. EMBO J 12: 4279-4290.

Naidu SR, Love IM, Imbalzano AN, Grossman SR, Androphy EJ. 2009. The SWI/SNF chromatin remodeling subunit BRG1 is a critical regulator of p53 necessary for proliferation of malignant cells. Oncogene 28: 2492-2501.

Narlikar GJ, Sundaramoorthy R, Owen-Hughes T. 2013. Mechanisms and functions of ATP-dependent chromatin-remodeling enzymes. Cell 154: $490-503$.

Nasipak BT, Padilla-Benavides T, Green KM, Leszyk JD, Mao W, Konda S, Sif S, Shaffer SA, Ohkawa Y, Imbalzano AN. 2015. Opposing calcium-dependent signalling pathways control skeletal muscle differentiation by regulating a chromatin remodelling enzyme. Nat Commun 6: 7441.

Ni Z, Abou El Hassan M, Xu Z, Yu T, Bremner R. 2008. The chromatin-remodeling enzyme BRG1 coordinates CIITA induction through many interdependent distal enhancers. Nat Immunol 9: 785-793.

Nora EP, Lajoie BR, Schulz EG, Giorgetti L, Okamoto I, Servant N, Piolot T, van Berkum NL, Meisig J, Sedat J, et al. 2012. Spatial partitioning of the regulatory landscape of the X-inactivation centre. Nature 485: 381-385.

Nora EP, Dekker J, Heard E. 2013. Segmental folding of chromosomes: a basis for structural and regulatory chromosomal neighborhoods? Bioessays 35: 818-828.

Padilla-Benavides T, Nasipak BT, Imbalzano AN. 2015. Brg1 controls the expression of $\operatorname{Pax} 7$ to promote viability and proliferation of mouse primary myoblasts. J Cell Physiol 230: 2990-2997.

Poole LA, Zhao R, Glick GG, Lovejoy CA, Eischen CM, Cortez D. 2015. SMARCAL1 maintains telomere integrity during DNA replication. Proc Natl Acad Sci 112: 14864-14869.

Rada-Iglesias A, Bajpai R, Swigut T, Brugmann SA, Flynn RA, Wysocka J. 2011. A unique chromatin signature uncovers early developmental enhancers in humans. Nature 470: 279-283.

Reyes JC, Barra J, Muchardt C, Camus A, Babinet C, Yaniv M. 1998. Altered control of cellular proliferation in the absence of mammalian brahma (SNF2 $\alpha$ ). EMBO J 17: 6979-6991.

Ross-Innes CS, Brown GD, Carroll JS. 2011. A co-ordinated interaction between CTCF and ER in breast cancer cells. BMC Genomics 12: 593. 
Sala A, Toto M, Pinello L, Gabriele A, Di Benedetto V, Ingrassia AM, Lo Bosco G, Di Gesù V, Giancarlo R, Corona DF. 2011. Genome-wide characterization of chromatin binding and nucleosome spacing activity of the nucleosome remodelling ATPase ISWI. EMBO J 30: 1766-1777.

Saladi SV, Keenen B, Marathe HG, Qi H, Chin KV, de la Serna IL. 2010. Modulation of extracellular matrix/adhesion molecule expression by BRG1 is associated with increased melanoma invasiveness. Mol Cancer 9: 280 .

Salma N, Xiao H, Mueller E, Imbalzano AN. 2004. Temporal recruitment of transcription factors and SWI/SNF chromatin-remodeling enzymes during adipogenic induction of the peroxisome proliferator-activated receptor $\gamma$ nuclear hormone receptor. Mol Cell Biol 24: 4651-4663.

Sanyal A, Lajoie BR, Jain G, Dekker J. 2012. The long-range interaction landscape of gene promoters. Nature 489: 109-113.

Scheibe M, Arnoult N, Kappei D, Buchholz F, Decottignies A, Butter F, Mann M. 2013. Quantitative interaction screen of telomeric repeat-containing RNA reveals novel TERRA regulators. Genome Res 23: 2149-2157.

Sehgal N, Seifert B, Ding H, Chen Z, Stojkovic B, Bhattacharya S, Xu J, Berezney R. 2016. Reorganization of the interchromosomal network during keratinocyte differentiation. Chromosoma 125: 389-403.

Seitan VC, Faure AJ, Zhan Y, McCord RP, Lajoie BR, Ing-Simmons E, Lenhard B, Giorgetti L, Heard E, Fisher AG, et al. 2013. Cohesin-based chromatin interactions enable regulated gene expression within preexisting architectural compartments. Genome Res 23: 2066-2077.

Shain AH, Pollack JR. 2013. The spectrum of SWI/SNF mutations, ubiquitous in human cancers. PLoS One 8: e55119.

Shi J, Whyte WA, Zepeda-Mendoza CJ, Milazzo JP, Shen C, Roe JS, Minder JL, Mercan F, Wang E, Eckersley-Maslin MA, et al. 2013. Role of SWI/ SNF in acute leukemia maintenance and enhancer-mediated $M y c$ regulation. Genes Dev 27: 2648-2662.

Smith EM, Lajoie BR, Jain G, Dekker J. 2016. Invariant TAD boundaries constrain cell-type-specific looping interactions between promoters and distal elements around the CFTR locus. Am J Hum Genet 98: 185-201.

Stankunas K, Hang CT, Tsun ZY, Chen H, Lee NV, Wu JI, Shang C, Bayle JH, Shou W, Iruela-Arispe ML, et al. 2008. Endocardial Brg1 represses ADAMTS1 to maintain the microenvironment for myocardial morphogenesis. Dev Cell 14: 298-311.

Stavreva DA, Coulon A, Baek S, Sung MH, John S, Stixova L, Tesikova M, Hakim O, Miranda T, Hawkins M, et al. 2015. Dynamics of chromatin accessibility and long-range interactions in response to glucocorticoid pulsing. Genome Res 25: 845-857.

Symmons O, Uslu VV, Tsujimura T, Ruf S, Nassari S, Schwarzer W, Ettwiller L, Spitz F. 2014. Functional and topological characteristics of mammalian regulatory domains. Genome Res 24: 390-400.

Tang Z, Luo OJ, Li X, Zheng M, Zhu JJ, Szalaj P, Trzaskoma P, Magalska A, Wlodarczyk J, Ruszczycki B, et al. 2015. CTCF-mediated human 3D genome architecture reveals chromatin topology for transcription. Cell 163: $1611-1627$.

Therizols P, Illingworth RS, Courilleau C, Boyle S, Wood AJ, Bickmore WA 2014. Chromatin decondensation is sufficient to alter nuclear organization in embryonic stem cells. Science 346: 1238-1242.

Thompson K, Marquez SB, Lu L, Reisman D. 2015. Induction of functional Brm protein from Brm knockout mice. Oncoscience 2: 349-361.

Tolstorukov MY, Sansam CG, Lu P, Koellhoffer EC, Helming KC, Alver BH, Tillman EJ, Evans JA, Wilson BG, Park PJ, et al. 2013. Swi/Snf chromatin remodeling/tumor suppressor complex establishes nucleosome occupancy at target promoters. Proc Natl Acad Sci 110: 10165-10170.

Tomar RS, Zheng S, Brunke-Reese D, Wolcott HN, Reese JC. 2008. Yeast Rap1 contributes to genomic integrity by activating DNA damage repair genes. EMBO J 27: 1575-1584
Trapnell C, Hendrickson DG, Sauvageau M, Goff L, Rinn JL, Pachter L. 2013 Differential analysis of gene regulation at transcript resolution with RNA-seq. Nat Biotechnol 31: 46-53.

Trotter KW, Archer TK. 2008. The BRG1 transcriptional coregulator. Nucl Recept Signal 6: e004.

Van Bortle K, Nichols MH, Li L, Ong CT, Takenaka N, Qin ZS, Corces VG. 2014. Insulator function and topological domain border strength scale with architectural protein occupancy. Genome Biol 15: R82.

Varga-Weisz P. 2001. ATP-dependent chromatin remodeling factors: nucleosome shufflers with many missions. Oncogene 20: 3076-3085.

Vietri Rudan M, Barrington C, Henderson S, Ernst C, Odom DT, Tanay A, Hadjur S. 2015. Comparative Hi-C reveals that CTCF underlies evolution of chromosomal domain architecture. Cell Rep 10: 1297-1309.

Wang D, Sul HS. 1997. Upstream stimulatory factor binding to the E-box at -65 is required for insulin regulation of the fatty acid synthase promoter. I Biol Chem 272: 26367-26374.

Wang W, Côté J, Xue Y, Zhou S, Khavari PA, Biggar SR, Muchardt C, Kalpana GV, Goff SP, Yaniv M, et al. 1996. Purification and biochemical heterogeneity of the mammalian SWI-SNF complex. EMBO J 15: 5370-5382.

Wang N, Tytell JD, Ingber DE. 2009. Mechanotransduction at a distance: mechanically coupling the extracellular matrix with the nucleus. Nat Rev Mol Cell Biol 10: 75-82.

Wang Y, Wong RH, Tang T, Hudak CS, Yang D, Duncan RE, Sul HS. 2013. Phosphorylation and recruitment of BAF60c in chromatin remodeling for lipogenesis in response to insulin. Mol Cell 49: 283-297.

Whyte WA, Orlando DA, Hnisz D, Abraham BJ, Lin CY, Kagey MH, Rahl PB Lee TI, Young RA. 2013. Master transcription factors and mediator establish super-enhancers at key cell identity genes. Cell 153: 307-319.

Wu S, Ge Y, Huang L, Liu H, Xue Y, Zhao Y. 2014. BRG1, the ATPase subunit of SWI/SNF chromatin remodeling complex, interacts with HDAC2 to modulate telomerase expression in human cancer cells. Cell Cycle 13: 2869-2878.

Wu Q, Madany P, Akech J, Dobson JR, Douthwright S, Browne G, Colby JL, Winter GE, Bradner JE, Pratap J, et al. 2015. The SWI/SNF ATPases are required for triple negative breast cancer cell proliferation. J Cell Physiol 230: $2683-2694$.

Xi Q, He W, Zhang XH, Le HV, Massagué J. 2008. Genome-wide impact of the BRG1 SWI/SNF chromatin remodeler on the transforming growth factor $\beta$ transcriptional program. J Biol Chem 283: 1146-1155.

Xu R, Spencer VA, Bissell MJ. 2007. Extracellular matrix-regulated gene expression requires cooperation of SWI/SNF and transcription factors. $J$ Biol Chem 282: 14992-14999.

Zhang Y, McCord RP, Ho YJ, Lajoie BR, Hildebrand DG, Simon AC, Becker MS, Alt FW, Dekker J. 2012. Spatial organization of the mouse genome and its role in recurrent chromosomal translocations. Cell 148: 908-921.

Zhang Z, Cao M, Chang CW, Wang C, Shi X, Zhan X, Birnbaum SG, Bezprozvanny I, Huber KM, Wu JI. 2015. Autism-associated chromatin regulator Brg1/SmarcA4 is required for synapse development and myocyte enhancer factor 2-mediated synapse remodeling. Mol Cell Biol 36: 70-83.

Zuin J, Dixon JR, van der Reijden MI, Ye Z, Kolovos P, Brouwer RW, van de Corput MP, van de Werken HJ, Knoch TA, van IJcken WF, et al. 2014. Cohesin and CTCF differentially affect chromatin architecture and gene expression in human cells. Proc Natl Acad Sci 111: 996-1001.

Received November 5, 2015; accepted in revised form July 8, 2016. 


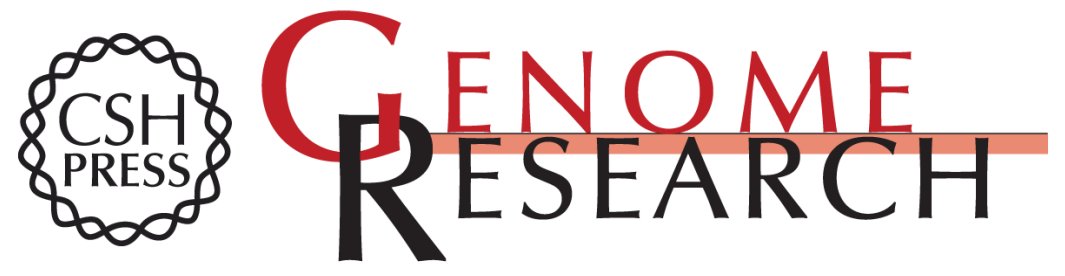

\section{SMARCA4 regulates gene expression and higher-order chromatin structure in proliferating mammary epithelial cells}

A. Rasim Barutcu, Bryan R. Lajoie, Andrew J. Fritz, et al.

Genome Res. 2016 26: 1188-1201 originally published online July 19, 2016

Access the most recent version at doi:10.1101/gr.201624.115

Supplemental Material

References

Creative

Commons

License

Email Alerting

Service
http://genome.cshlp.org/content/suppl/2016/08/09/gr.201624.115.DC1

This article cites 96 articles, 26 of which can be accessed free at: http://genome.cshlp.org/content/26/9/1188.full.html\#ref-list-1

This article is distributed exclusively by Cold Spring Harbor Laboratory Press for the first six months after the full-issue publication date (see

http://genome.cshlp.org/site/misc/terms.xhtml). After six months, it is available under a Creative Commons License (Attribution-NonCommercial 4.0 International), as described at http://creativecommons.org/licenses/by-nc/4.0/.

Receive free email alerts when new articles cite this article - sign up in the box at the top right corner of the article or click here.

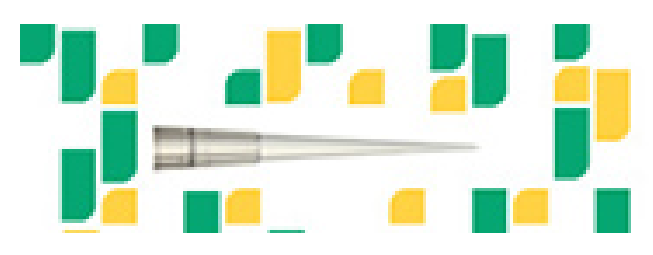

Focused on your science.

Jコగ

SCIENTIFIC

saos or seisnes

To subscribe to Genome Research go to:

https://genome.cshlp.org/subscriptions 